\title{
Computer Control of Large Accelerators Design Concepts and Methods
}

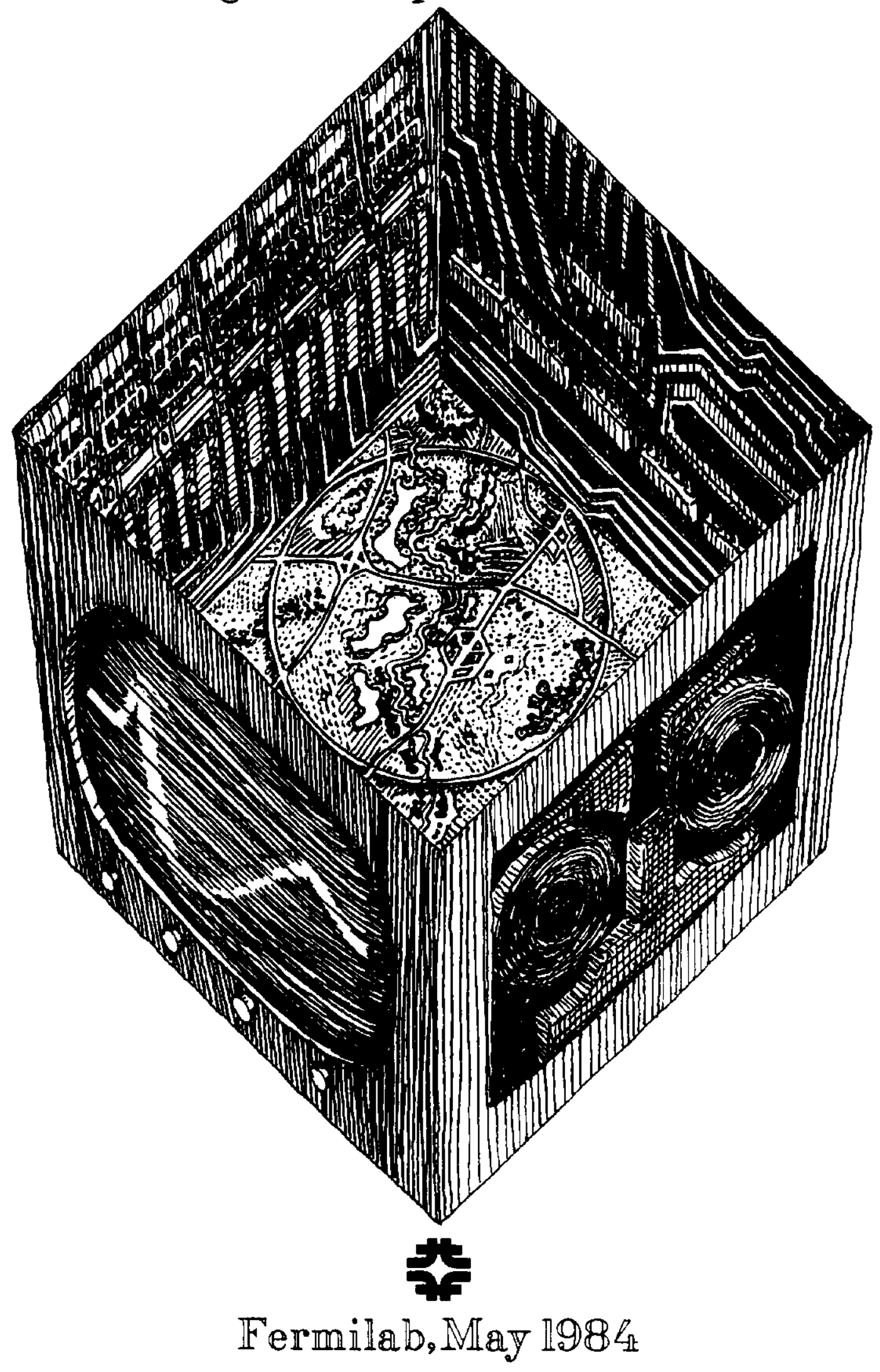


FERMILAB-Conf $-84 / 43$

0121.220

\title{
COMPUTER CONTROL OF LARGE ACCELERATORS
}

Design Concepts and Methads

\author{
F. Beck* and M. Gormley
}

Lectures given at

3rd Annual Summer School on High Energy Accelerators

July $6-16,1983$

BNL-Stony Brook

Long Island, New York

* On leave of absence from CERN. 
1. Computer Control Systems for Accelerators ............. 1-1

1.1 Introduction ............................. 1-1

1.2 What must the control system control? $\ldots \ldots \ldots \ldots \ldots \ldots 1-2$

1.3 Why distributed control? $\ldots \ldots \ldots \ldots \ldots \ldots \ldots \ldots \ldots \ldots$ 1-6

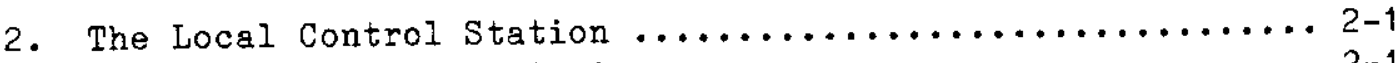

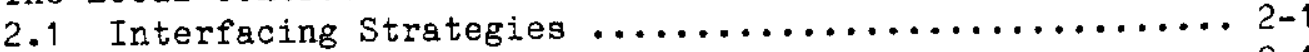

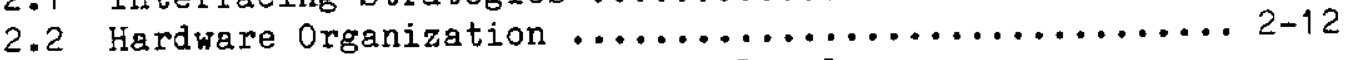

2.3 Interactive Facilities at the Local

Control Station ........................... 2-15

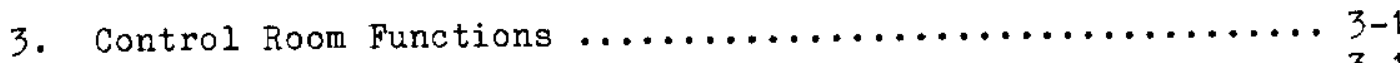

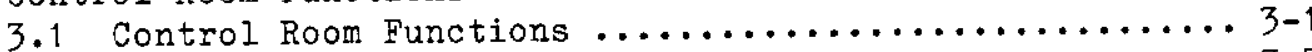

3.2 Control Programs ............................ $3-7$

3.3 Control Room Information Strategies ............ 3-11

4. Interconnection of the Local and Central stations ....... 4-1

4.1 Introduction ..........................

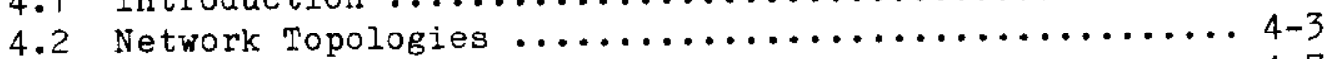

4.3 Access Methodologies ........................ 4-7

4.4 Encoding Schemes ............................ 4-13

4.5 Standards for Local Area Networks ................ 4-14

4.6 Application to Accelerator Control Systems ........ 4-16

5. Information Strategies ...................... 5-1

5.1 Types of Data Base ...................... $5-1$

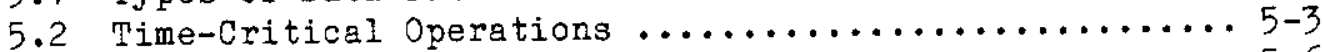

5.3 Protection Requirements ................... 5-6

5.4 Pulse-to-pulse Modulation .................... 5-10

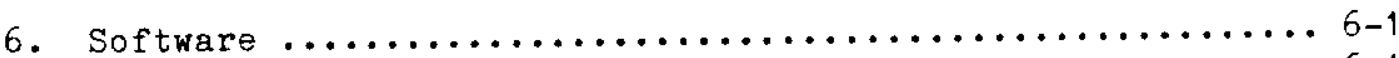

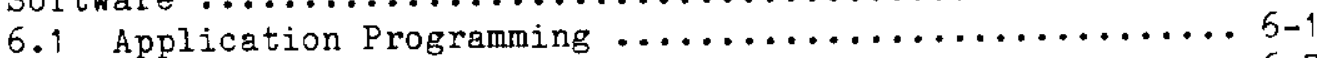

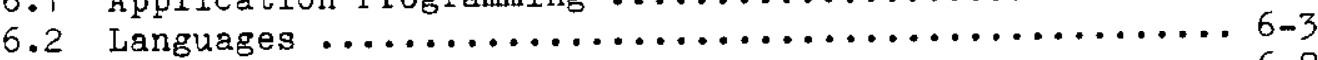

6.3 Aids to Understanding $\ldots \ldots \ldots \ldots \ldots \ldots \ldots \ldots \ldots \ldots \ldots .6 .6$

6.4 Programming the Microprocessors ...............6-9

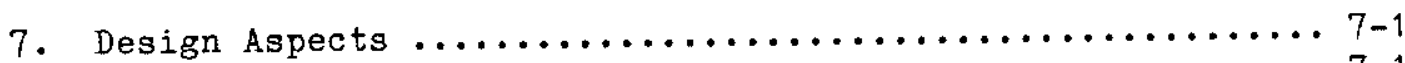

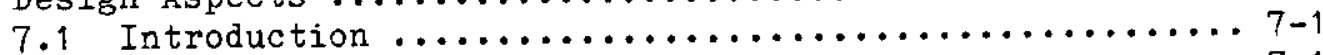

7.2 Costs: Where Does the Money Go? ............... 7-1

7.3 Planning and the Setting of Time-Scales .......... $7-5$

7.4 Open-ended Design .......................... $7-6$

Problem $1 \ldots \ldots \ldots \ldots \ldots \ldots \ldots \ldots \ldots \ldots \ldots \ldots \ldots \ldots \ldots \ldots \ldots \ldots$

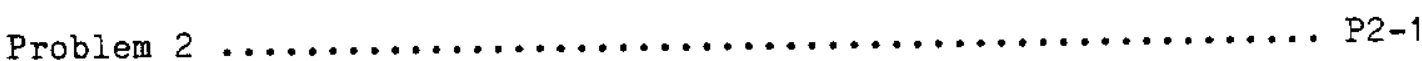

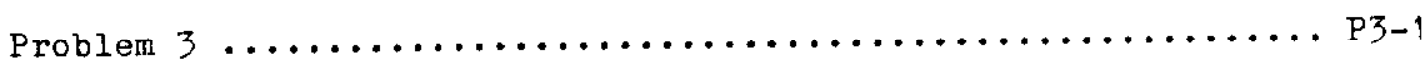

References $\ldots \ldots \ldots \ldots \ldots \ldots \ldots \ldots \ldots \ldots \ldots \ldots \ldots \ldots \ldots \ldots \ldots \ldots$ 


\begin{abstract}
Unlike most of the specialities treated in this volume, control system design is still an art, not a science. These lectures are an attempt to produce a primer for prospective practitioners of this art.

A large modern accelerator requires a comprehensive control system for commissioning, machine studies and day-to-day operation. Faced with the requirement to design a control system for such a machine, the control system architect has a bewildering array of technical devices and techniques at his disposal, and it is our aim in the following chapters to lead him through the characteristics of the problems he will have to face and the practical alternatives available for solving them.

We emphasize good system architecture using commercially available hardware and software components, but in addition we discuss the actual control strategies which are to be implemented since it is at the point of deciding what facilities shall be available that the complexity of the control system and its cost are implicitly decided.
\end{abstract}


CHAPTER 1

COMPUTER CONTROL SYSTEMS FOR ACCELERATORS

\subsection{Introduction}

The inclusion of material on computer control in this series of lectures is a new departure, and it might be useful to digress for a moment on the aim of the exercise, before launching forth on the material proper.

The design of control systems for accelerators has in the past been a sort of afterthought, neglected in the first flush of excitement in favor of the more glamorous fields of magnet design and beam physics. A proposal for a large new machine often contains a global price estimate for control, and no further mention of it. Discussion of the design problems of control systems might therefore be a useful move, if only to increase awareness in accelerator builders of the need to incorporate control-system ideas at the earliest stage of the conception of a new machine.

It must be stated straightaway that it is to the design of control systems for large machines that we turn our attention here. Whilst the principles of good design are of course universal, it is not until the machine grows beyond the need for more than one computer that the problem becomes non-trivial. With a home computer and one of the available crate-and-bus systems, it is possible to produce a very satisfactory control system for a small machine, and the impression of complete mastery of the problem will prevail until the system is called upon to expand or to liaise with another control system, such as that employed by the experimenters using the machine, or that installed by the builders of a second accelerator, for which the first is to act as injector. 
In addition, there is a considerable difference between the requirements for a machine operated by its builders on an informal basis, and those for a large complex which will be served by teams of professional operators. A control system for such a machine must not only be "operator friendly", but it must also allow for the transfer of expertise from the builders to the users. It is also a fact that a large complex, having many more degrees of freedom, will attract skilled innovators who will repeatedly suggest and implement changes in the structure and mode of operation of the accelerator complex.

Finally, there is the inescapable fact that accelerators are very expensive installations, and are therefore amortized over a very long period. Twenty years is not atypical, and there are several accelerators in use twenty-five years after their original commissioning. Control systems, on the other hand, use the technology of electronics and computer science. This technology has been advancing at such a pace in recent years that it is often cheaper to replace something than to improve it, and none of the twenty-year old complexes mentioned above has been able to escape the need to rebuild its control system at least once.? We now have available sufficient hindsight to enable us to design control systems with expansion and rebuilding in mind.

There are important factors of even this limited problem which we do not intend to cover here. Not only are programming, electronic design and computer architecture important considerations in the design of computer control systems, but they would lend themselves well to the structure and form of this school. Tutorial sessions followed by exercises would follow these subjects through systematically. Such an approach, however, would not only cover well-explored territory for which guides are available elsewhere, but would omit an important area which has been less well documented, namely that of the principles and the criteria which need to be considered when planning the control for a large machine.

To sum up this introduction, therefore, we will discuss here only control principles, and those only for large, expensive accelerator complexes, which are likely to be operated by people other than the designers, and which represent such a large capital investment that they are likely to have a useful lifetime, probably in much modified form, of many years.

1.2 What must the control system control?

Apart from the vacuum chamber carrying the beam, the control system is the only element of the accelerator which 
links all its major components, and most subsystems require some service from it. When computing was expensive, it was only used to perform operations not possible otherwise. Nowadays it forms the cheapest and most convenient methodology for doing numerous less vital housekeeping tasks around the machine, as well as the more conventional calculations. Even the advent of localized computer control does not obviate the need for the services of a more generalized system to guide and monitor the local process.

What then are the subsystems with which we are concerned? In the next few paragraphs we ennumerate them:

\subsubsection{Power Supplies}

Steering and focusing are done by magnets, and in many cases these are fed by pulsed power supplies whose output is carefully controlled. There are many aspects of this control which need the computer system. Generation and distribution of the time functions, often entailing learning processes which use global input, control and surveillance of the individual supplies, and overviewing of the personnel protection arrangements can all be done in this way.

\subsubsection{Cryogenic Systems}

When the ragnets use superconducting technology, there are many chores which the computer system can pick up. Quite apart from the control of the industrial-type plant producing and distributing the liquid helium, there is the more critical task of detecting incipient quenches and containing their consequences. It might be thought that a task of such importance for the safety of man and machine is too critical to be trusted to a computer, but provided the system is kept conceptually simple it transpires that this is the only practicable way to do it properly. ${ }^{2}$ In addition to the quench protection mechanism itself, there is of course a need for diagnostic and post-morten facilities to monitor it.

\section{2 .3 RF Plant}

The RF plant, in a way, is the accelerator. Much of the control work here is by feedback loops in the nanosecond range, and uses the techniques of microwave technology and analog engineering. 3 Nevertheless, more and more of this material needs to be subjected to computer interfacing, so that work traditionally relegated to knob twiddlers and dial watchers can be done remotely or even included in slow closed loops. This 
tendency is reinforced by the fact that many accelerators deliver beam alternately to more than one user, and that this may entail periodic changes in the RF parameters, preferably under computer control. Similarly, the RF gymnastics for bunching and debunching necessary on hadron accelerators render computer control of the parameters a necessity.

The other aspect of computer control for the RF system is of course that it, like many other subsystems, involves a large complex of power supply, cooling and other services which need to be monitored and controlled.

\subsubsection{Beam instrumentation}

These large auchines cannot be operated without proper instrumentation, and such instrumentation requires that the information be made available on a global basis to whoever is tuning or operating the machine. The position and profile of the beam all round the machine can be measured by a variety of devices, some of which even need local control at each measurement station. 4 This can result in a large quantity of information which must be conveyed to some central place in order to process or display it on a global basis. In addition beam current can be measured and its time structure dispiayed and analysed. Beam losses can be measured, both "on the fly" and in integrated form. A more informal view of the particle beam is often provided with flags or screens moved temporarily into its path. In this case, remote control of the mechanical device and of the TV cameras used for the observation are an integral part of the requirement.

\subsubsection{Beam line and Switchyard control}

This subsystem really embodies many of the requirements outlined for the others listed above, but the operational requirements are more specialized. In order to tune a beamline it is obviously necessary to set its steering and correction elements and to determine the path and profile of beam, but it is also necessary to do this repeatedly and to restore quickly operating styles which have been set up and stored previously. The control system must export data about the current machine performance to experimenters and their computers, and it may be desirable for the experimenters to have control of their own secondary beams. At this point the control of the accelerator and the instrumentation of the experiment merge imperceptibly, and the advantages of homogeneous systems become evident. The separation of 
responsibilities becomes even more hazy in the case of experiments using colliding beams or gas jets, where the experiment is actually situated in the accelerator itself. Strangely enough, there is no laboratory known to the authors where control of the accelerator is managed by the same people as control of the experimental areas, or even where the same techniques are used for both.

1.2.6 Machine Physics

Since the systems described up to this point can be used to look at and influence all components for which instrumentation and control are provided, and since all this information is available in a computer system, one might think that the needs of the machine physicist are answered in the same way as those of the operator. This is not so. The display of profiles, time-plots and phase space diagrams is certainly necessary, but it is not sufficient. Machine Physics, by its very nature, makes unpredictable demands on the control system. It must be easy to incorporate new or experimental instrumentation and to write tentative programs for new modes of operation. An accelerator is not an industrial plant, with a fixed regimen of speration. It is a living organism, constantly being adjusted, corrected and improved, and with the best of these adjustments being incorporated permanently into the structure. A control system which is unable to keep up with this requirement for continuous amendment may actually hamper the development of the machine for which it is designed, and thus eventually limit its usefulness as an instrument for physics.

\subsection{Miscellaneous Services}

'Ne have purposely reserved mention of these services until last. They comprise the control of the vacuum system, the surveillance of radiation protection and personnel access, and the supply of the various services which are vital to the complex. These miscellaneous services add a negligible load to the control system, and provide few profound technical problems. Nevertheless, it is impossible to control a machine which physically covers an area of several square miles without an efficient system of remote control and surveillance. In general the telemetry and remote control of electricity, fluids and ventilation circuits does not impose design constraints on a system which is sufficiently powerful and flexible to satisfy the other technical requirements. 5 


\subsection{Why distributed control?}

From the next chapter on, we shall make the tacit assumption that a control system of this kind should have a "distributed" design both with respect to the location of the intelligent components and to access by people. In the past, all control systems were centralized, by virtue of the enormous cost of a computer. Now that distribution is possible, the designer has a choice. New is not necessarily better. Let us look at the possibilities:

1.3.1 Central computers with dumb interfaces

This is the original method for building a control system. The interfaces contain simple or at most buffered registers, and the single computer performs the threefold sevice of data collection, processing and human interface management.

1.3.2 Several computers with dumb interfaces

When the single computer begins to overload, there
is no alternative to adding another. This implies
completely separate systems for different parts of the
equipment, with separate operator interfaces and
independent calculation facilities. This is sometines
practicable when the equipment separates naturally into
operationally disparate elements, as in a Linear
Accelerator, Booster and Main Ring.

1.3.3 Intelligent interfaces

Microcomputers enable many operations to be performed in the hardware interface itself. This arrangement not only relieves the computer of some work, but glso allows bandwidth reduction, reducing the flow of information into and out of the computer. A typical example is a function generator, which produces a repetitive waveform for ever when once given the coordinates of the curve to be generated. The very high information flow needed by graphics devices may be reduced by such means, and $a$ ball and cursor may be connected without placing any load on the main computer until it is required to trangmit the coordinates for control purposes.

\subsubsection{Full distribution}

We note that the process of subdivision brings incidental advantages. A system consisting of many intelligent elements allows easy expansibility, the 
possibility of redundant arrangements, program simplicity in each unit and lower information transfer rates across the system.

The one disadvantage of a distributed system, the inability to combine information from several subsystems in a global process, can be obviated by the use of a connecting network. The following chapters will therefore discuss, not only the individual elements needed for the design of a distributed system, but also the information strategies which can make an integrated whole out of a number of otherwise independent subsystems. 
CHAPTER 2

THE LOCAL CONTROL STATION

In summarizing and reviewing the repertoire of electronic interfacing strategies, we emphasize the environment of a geographically large system - one where the interface electronics are situated in discrete locations which are separated from each other by large distances (50 meters or more) and which are remote from the central control room. We use the phrase "the local control station" to mean that area where input signals are conditioned and converted from analog to digital form and where output signals are converted from digital representations to analog levels.

\subsection{Interfacing Strategies}

Over the last twenty years the elementary building blocks for interfacing a diverse assortment of hardware to a computer-based control system have remained essentially the same: (multiplexed) analog-to-digital converters, binary input modules, (multiplexed) digital-to-analog converters, binary output modules and interrupt facilities. However, the design of the local control station and the organization of its hardware components have changed dramatically over the same period. Throughout the decade of the 1960's, electronic interfacing strategies relied exclusively upon in-house designs which organized and packaged the components in customized "boxes". The disadvantages of this approach for control system applications are plentiful: it is uneconomical both in dollars and manpower; it tends to be non-modular; it is inherently non-extensible; and so on. To overcome these disadvantages the concept of an instrumentation bus appears to have evolved in the late 1960's. Initial efforts on 
designing, developing and using instrumentation busses were, again, customized, in-house approaches. Not until the early 1970 's was it recognized that standards needed to be established for instrumentation busses. A standard, in this context, is a model or pattern laid down by some authority by which the quality or correctness of a thing may be determined.

The authorities which initiated the efforts of developing instrumentation bus standards were the ESONE Committee of European Laboratories, the NIM Committee of the U. S. Energy Research and Development Administration, the Institute of Electrical and Electronic Engineers (IEEE), the International Electrotechnical Commission (IEC), and the American National Standards Institute (ANSI). Over the last decade these organizations have adopted and endorsed a wide variety of instrumentation bus standards. Most of these standards specify both the mechanical and electrical characteristics of the interface hardware as well as the set of interfacing signals and the ground rules for using them.

Some of the interface standards employ a dataway which is designed to be managed by a single master control station: all secondary stations on the dataway are slave stations. other standards employ more democratic and flexible dataway designs in which any station on the dataway can become bus master. Some of these standards use only $t 0$ to 20 signal lines and rather modest-sized printed circuit boards. Others employ a hundred or more signal lines and huge, multi-layered, printed circuit boards. There is an obvious correlation between the size of a printed circuit board, its costs and what it will do. The control system designer needs to understand the performance requirements of the interface hardware in order to select an instrumentation bus that will provide the necessary capabilities in a cost-effective manner.

2.1.: The General Purpose Interface Bus, GPIB (IEEE 488)

The first instrumentation bus approved by the IEEE Standards Board was IEEE 488 - 1975: "Digital Interface for Programmable Instrumentation", more commonly called GPIB. 6 This particular scheme, developed at Hewlett-Packard and sometimes called HPIB, uses 16 signal lines for interconnecting all the devices on the bus in parallel. The signal lines are usually bussed from one device to another using a passive, multi-conductor cable 
which groups the signal lines into three clusters: Data Bus ( 8 lines), Transfer Control Bus ( 3 lines), and Management Bus ( 5 lines). The 8-bit Data Bus is used to carry addresses, data, commands, and status to and from devices in a bit parallel, byte serial format.

The availability of only 16 signal lines implies that the IEEE 488 instrumentation bus has serious inadequacies that limit its use in a large accelerator control system. It is a common criticism that its length and the number of loads it supports are inadequate. 7 However, this bus has been well accepted by instrument manufacturers: more than 1500 products using the IEEE 488 bus are available today from more than 250 vendors. As a result, there exists a large collection of complex, programmable instrumentation (spectrum analyzers, frequency synthesizers, etc.) which is quite often attractive to accommodate in an accelerator control system and for which the IEEE 488 bus is a de facto standard.

The design of the local control station for any new, large accelerator complex should accommodate the ability to connect specialized, complex, programmable instruments which use the IEEE 488 standard; but the IEEE 488 bus should not be the primary strategy used for interfacing the bulk of the equipment.

2.1.2 The CAMAC Instrumentation Bus (IEEE 583)

The instrumentation bus used within the CAMAC system (Computer Automated Measurement and Control) was the second one to be approved by the IEEE Standards Board.8 Its advantages of modularity, speed ( 1 microsecond dataway cycle time), international availability, etc. are well known to both the high energy physics community and to the accelerator control system designer. By the middle of the 1970 's CAMAC had established itself as the prevalent instrumentation bus for accelerator control systems. Systems with large numbers of CAMAC crates, linked by various parallel or serial highway arrangements, are in common use today.

The introduction in 1974 of powerful, inexpensive microprocessor components had a profound and rapid impact on all instrumentation systems. The impact on 
CAMAC-based systems was both pervasive and confusing to the system designer. Some designers began to distribute monitoring and control functions by incorporating intelligent CAMAC modules into their systems; others interfaced complete, commercial microsystems to modules which resided on the CAMAC dataway; intelligent crate controllers were invented for stand-alone operation or for consolidating communications between a host computer and the modules in a crate; the CAMAC standards committees found it desirable to invent and specify an auxiliary controller bus to provide multiple sources of control over the modules in a single crate (IEEE 675).

All large accelerator control systems have found it both necessary and desirable to minimize the degree of centralized monitoring and control of devices by distributing these functions to the local control station. The diverse approaches toward embedding intelligence in CAMAC-based systems were usually quite awkward and complicated.9

The most natural and effective method of incorporating intelligence into the electronics at the local control station is to employ a bus structure that can serve simultaneously both as a processor bus and an instrumentation bus. One retains the same high level of modularity that one enjoyed with CAMAC-based systems because a repertoire of instrumentation modules can be plugged into the bus. In addition, a flexible collection of processor-oriented modules (memory, communications controllers, etc.) can be supported off the same bus to produce a highly modular and flexible configuration that can be matched to the needs of the application. Ideally, the processor's access to the instrumentation hardware becomes as easy as a memory access. The necessity of incorporating intelligence into the local control station and the availability of powerful yet inexpensive microprocessor components have resulted in the CAMAC dataway being gradually supplanted by the processor-oriented busses in large accelerator control systems.

2.4.3 The STD Bus (IEEE P961)

The lowest performance system bus that is reasonably well supported by commercial vendors is the STD bus, introduced by Mostek and Prolog in 1978.10 It employs an 
8-bit data path and a separate 16-bit address path. STD boards measure $4.5^{\prime \prime}$ by $6.5^{\prime \prime}$ and are available from more than 70 different companies. Figure 2.1 shows a typical STD board.

Currently the STD bus appears to be enjoying a renaissance of renewed interest from designers as a result of the fact that VLSI technology fits more functions onto a single chip, thereby allowing board-level designers to support more complex functions in smaller packages. The original specification, however, made no provision for the arbitration of simultaneous bus requests and, consequently, did not support multiple processors or the presence of more than one DMA-based card in the same crate.

The STD manufacturers' committee has proposed arbitration schemes to eliminate these restrictions and steps are being taken to formalize the bus specifications as IEEE P961. A standardization effort for an STD-like bus with a Eurocard format, called STE, is also under development (IEEE P1000). The STD bus is explicitly intended for use with 8-bit processors and, as a result, is limited and restricted to applications with low performance requirements.

The control system designer might well find the STD bus to be adequate if his application is extremely well-defined and has low performance requirements. On the other hand, applications with moderate performance requirements or environments in which there is a high premium on expansion capability and the ability to upgrade, would not be well suited to the use of the STD bus.

\subsubsection{S-100 Bus (IEEE 696)}

The evolution of the $S-100$ microcomputer bus is interesting because it demonstrates all the pitfalls that can occur when a design technique becomes a popular convention without being formally specified by a recognized authority. The $S-100$ bus was developed as the motherboard for the Altair 8800 microcomputer, an immensely popular hobbyist's system. The design, published in Popular Electronics in 1975 employed a 100-pin connector to interconnect all cards and by the late $1970^{\prime} \mathrm{s}$ several hundred vendors were producing $\mathrm{S}-100$ modules. The design was sufficiently well defined that 


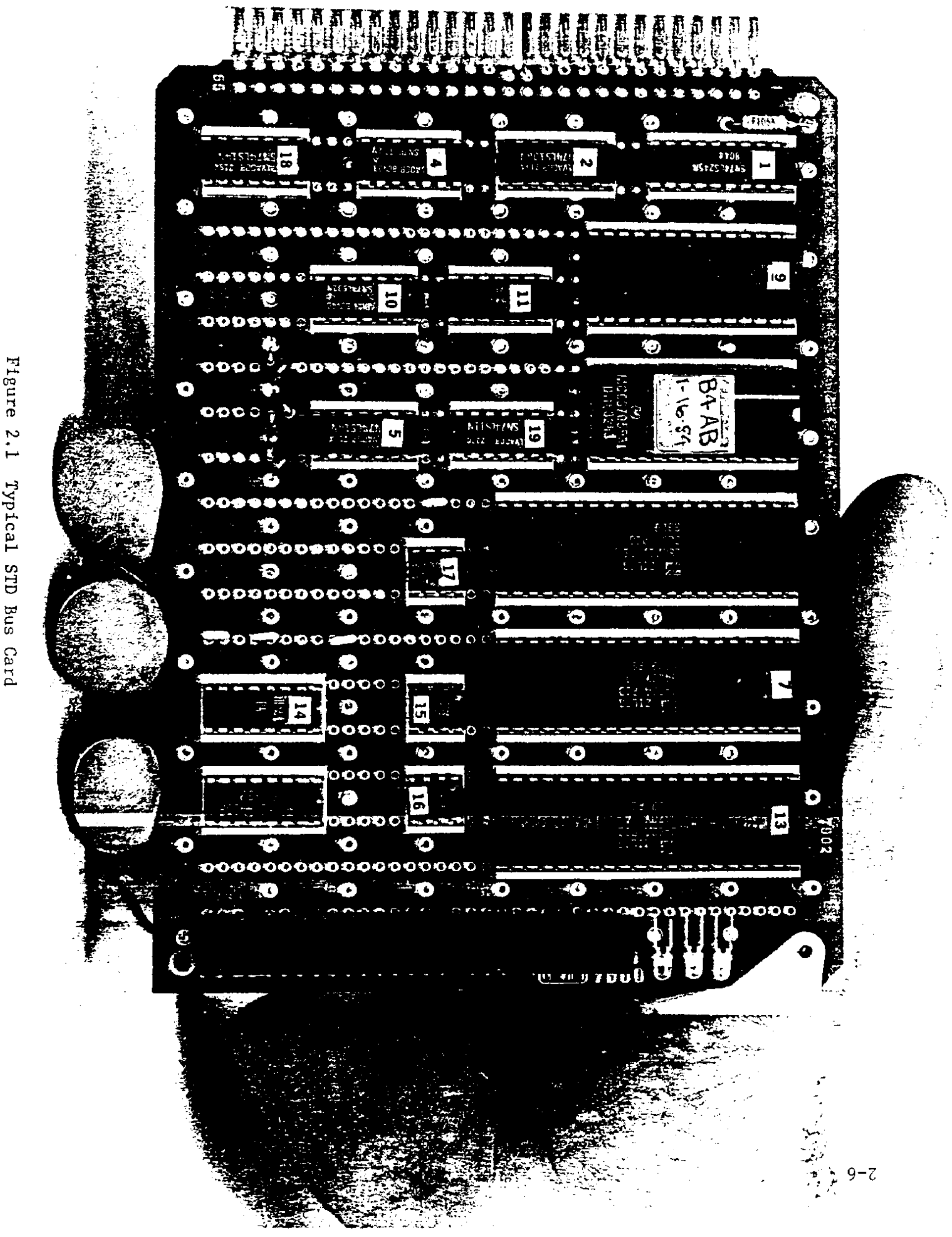


most modules ordinarily worked well together, but the specification was sufficiently incomplete that a large number of modules emerged which were incompatible because they made conflicting uses of some of the bus lines, e.g., the spare pins.11 Since the original manufacturer never published a specification, he retained the freedom to change pin designations or allocate spare pins as he saw fit. The chaos created by this lack of specification became legendary. The IEEE managed to completely specify the bus and adopted it as a standard system bus in 1982 (IEEE 696).12 However, updating the bus and making it conform to IEEE specifications has produced additional problems, for the short term at least, since the marketplace now offers both non-standard as well as standard $S-100$ boards.

S-100 based systems offer performance that is comparable to that of Multibus systems (see below) but a much smaller collection of process control instrumentation modules is available for the $5-100$ bus. For control system applications the Multibus is the preferred system for satisfying medium performance requirements.

2.1.5 Multibus (IEEE 796)

The most prevalent medium performance system bus in use today is the Multibus, introduced by Intel in 1973 and approved as IEEE Standard 796. in 1982.13 It contains 20-24 address lines, 16 data lines and 8 interrupt lines. Figure 2.2 shows a typical 6.75 " by 12 " Multibus card.

The Multibus is well supported by commercial vendors: a variety of both 8-bit and 16-bit processor boards and a choice of a large number of memory configurations is available. The repertoire of process control instrumentation modules that are designed for use with the Multibus is quite extensive. In addition, bus arbitration is provided for the use of multiple masters and the bus itself can be constructed using modular backplane units which allow the number of slots in the crate and the size of the system to be tailored to the complexity of the application.

As a system bus, the Multibus satisfies all the requirements of most control system applications: it offers moderate performance, is well supported by the 


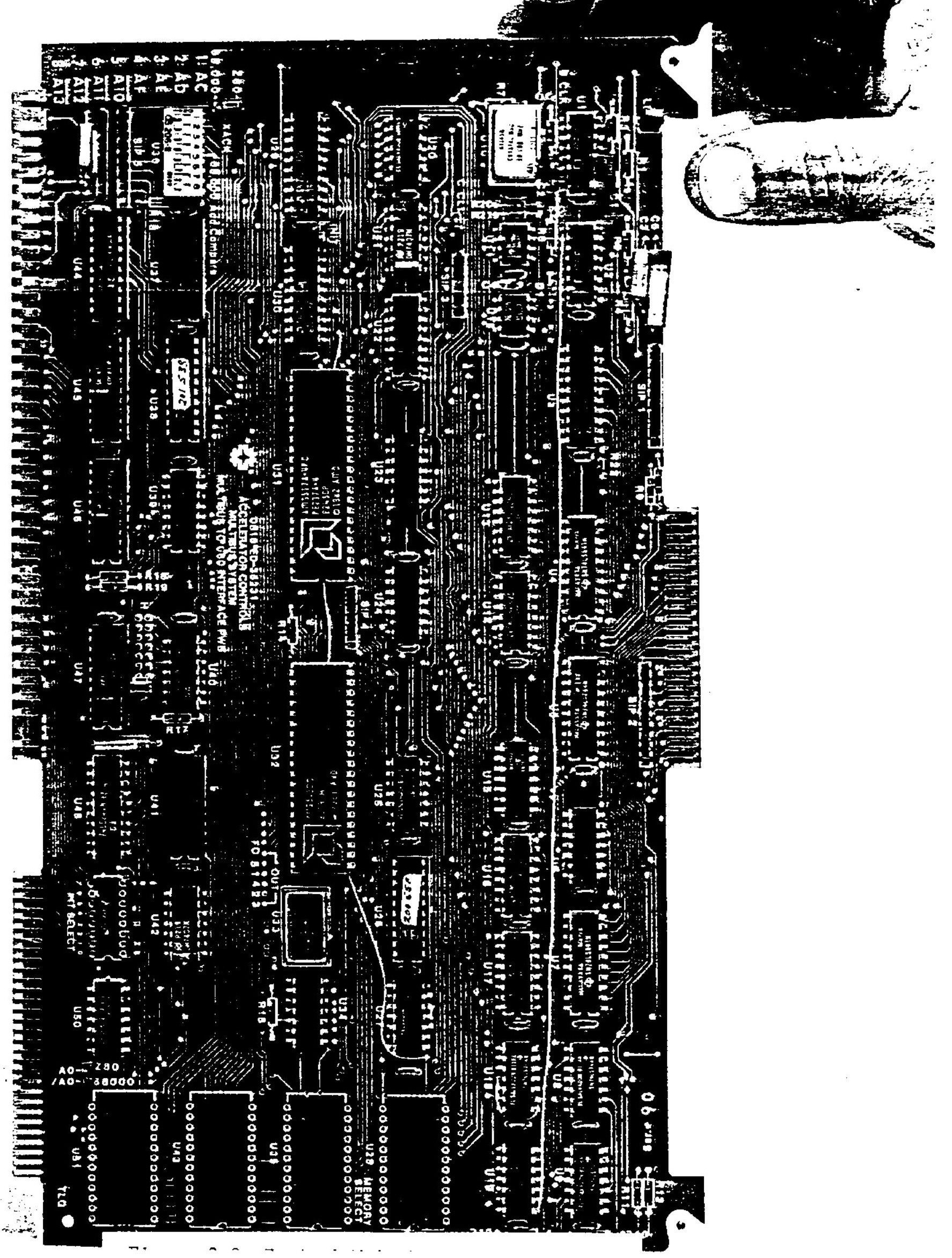


commercial marketplace, can be fairly well configured to the application at hand, makes provision for multiple mastership of the bus and offers reasonable expansion capability.

\subsubsection{FASTBUS (IEEE P960)}

The development of FASTBUS began in 1975 under the auspices of the NIM and ESONE Committees. The motivation, driving this development work, has been the need for a system that will meet the high speed data acquisition and parallel processing requirements of the next generation of large-scale high energy physics experiments. Consequently one of the primary goals of the project has been to develop a system with the highest possible throughput - at least an order of magnitude bandwidth improvement over CAMAC. 14

The first complete set of FASTBUS specifications, published in November, 1982 and proposed as specification IEEE-P960, describes two different types of crates and several different methods for interconnecting them.15 A single-width FASTBUS module, shown in Figure 2.3, is 14.44 " high by 15.75 " deep (367 mm. by $400 \mathrm{~mm}$.) and mates to the crate dataway with a 130 pin connector. The dataway itself is typically implemented as an eight layer printed circuit board which supports a 32-bit multiplexed address and data bus. Any module in the crate is capable of becoming a bus master. Single 32-bit transfers are completed in about 100 nanoseconds using a handshaking convention; block transfers between modules in the same crate are roughly twice as fast.

One interesting characteristic of FASTBUS is its concept of bus segmentation. When a module becomes bus master, it can communicate with other modules in the same crate or in other crates using the same protocol. This characteristic makes it possible to devise rather flexible and complex configurations that support distributed processing requirements which span several crates.

The exceptional high-speed throughput offered by FASTBUS does not, of course, come free of charge. Crate costs and module costs are higher than for any of the other bus systems reviewed here and the hardware interface between a module and the bus is significantly 

(1)

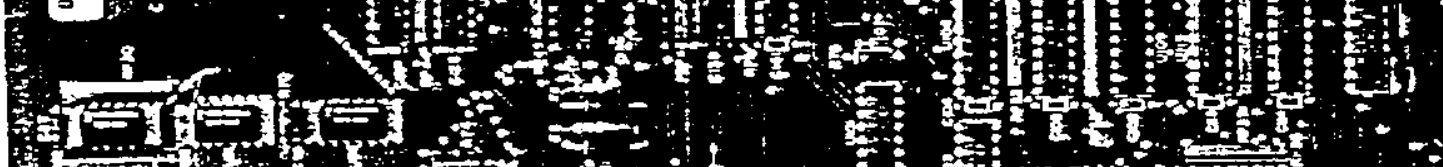
(1) ton (1)

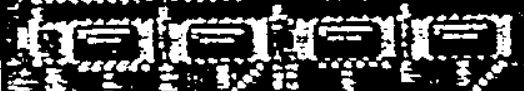
ㄱ.

$+2$ an $=10$

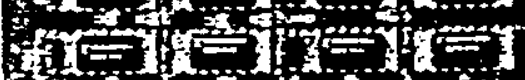
LU

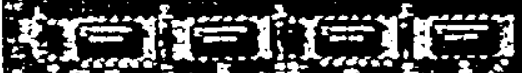

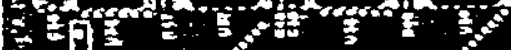
25

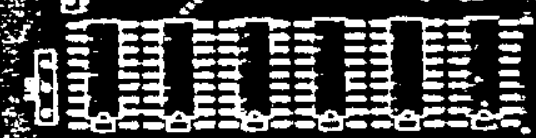

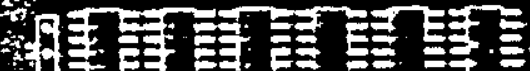

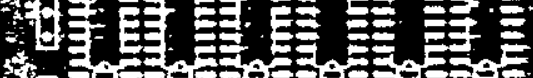

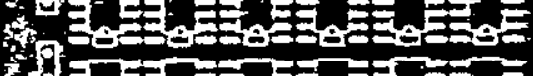

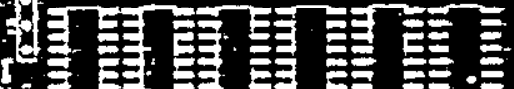
4.

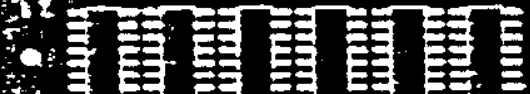
\%

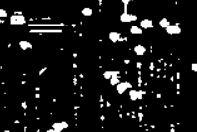

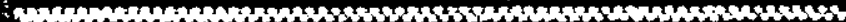
40 and

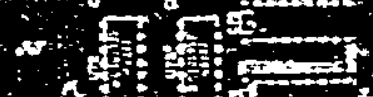

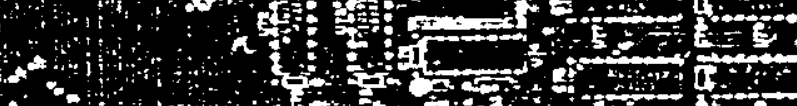
a 20 at 30 a 4 a

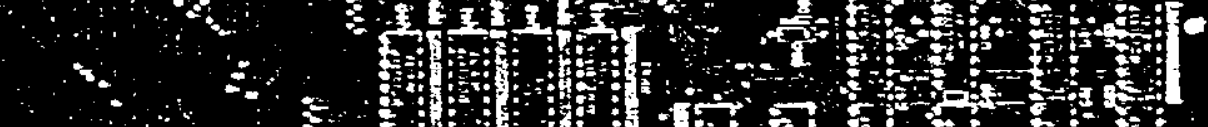

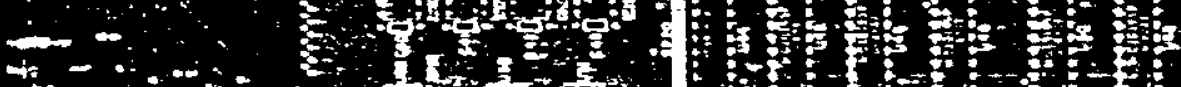
4 . If 5 o

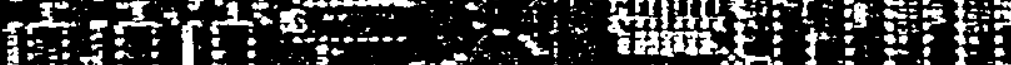

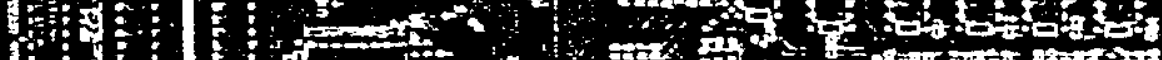
190150 d

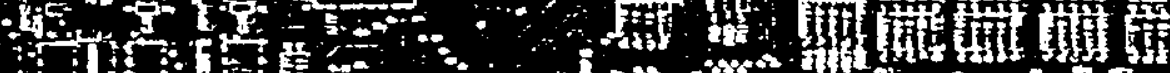
1. PItrom 
more complex. Although some of the developers of FASTBUS have advocated its use within control systems, we are not aware of any existing accelerator control system requirements that cannot b ${ }_{6}$ satisfied by less costly and less complex system busses.

\subsubsection{Advanced System Busses}

Most of the popular system busses in use today were designed and developed by manufacturers and became de facto standards even though they were usually incompletely specified. In an attempt to avoid this situation in the future, a working group of the IEEE Microprocessor Standards Committee began to examine future system bus requirements in 1979. Their major conclusion has been that the $S-100$ and Multibus busses could not be extended to satisfy the requirements anticipated for future microprocessor based systems. Consequently, they have proposed a standard for an Advanced Microcomputer System Backplane Bus (IEEE P896), which is ordinarily called Future Bus, and which is intended to satisfy, for at least 10 years, a full range of interconnection requirements for 16- and 32-bit microcomputers, frpm simple systems to multiprocessor, multitasking ones. After four years, however, the proposed specifications are still in the draft stage and no manufacturer has openly endorsed the specification.

In the interim, two fairly similar high performance system busses have been developed and announced by Motorola and its collaborators: the VERSAbus and the VME bus. Both are designed to support 32-bit as well as 16-bit processors and both make provision for multiple masters and slaves. The VME bus uses single ( $160 \times 100$ $\mathrm{mm}$ ) and double $(160 \times 233 \mathrm{~mm})$ Eurocard board sizes and is a joint development of Motorola, Mostek, Signetics/Philips and Thompson-CSF. The interest in accommodating the European marketplace is not surprising since it is estimated that the board computer market in Europe 18 accounts for around 40 percent of the world total. 18 Some of the performance characteristics and properties of the VME bus are somewhat staggering: it supports 32-bit, unmultiplexed data transfers and provides 24-32 address lines to pernit direct addressing of over 4 Gigabytes of storage. Whenever control system applications find they need very high performance hardware, it clearly will be a serious candidate. 


\subsection{Hardware Organization}

It is difficult to make specific and concrete recommendations on the hardware organization of the local control station's components that would have universal validity, $i$. e., that would apply to all categories of accelerator complexes. The geographical size of the accelerator, the nature and number of equipment enclosures or service buildings, the civil construction techniques used to house the accelerator itself (tunnel, hall or pipe) all influence both the selection and the organization of the hardware components used to implement the local control station. We restrict our discussion to a limited class of environments, specifically those for which:

1 The accelerator complex includes a circular machine with a diameter of at least $2 \mathrm{~km}$.

2. The magnet power supplies, interface electronics, etc. are located, in accessible equipment enclosures, outside the tunnel or pipe which contains the ring.

3. The equipment enclosures are, roughly, equally spaced around the ring.

4. The location of the equipment enclosures exploits the symmetry of the lattice and the allocation of equipment to the different enclosures is highly symmetrical from one enclosure to the next.

The major functional components of a machine in this category are well-known and can be divided into two groups: those that perform a localized function and are contained in a limited section of the ring (the injection system, the $r f$ acceleration system, and the extraction system, for example) and those which perform a global, or ring-wide, function which is distributed over the entire ring (the vacuum system, the main magnets, the correction element coils, the beam position and intensity detectors, the beam loss monitors, and if it is a superconducting ring, the cryogenic and quench protection systems). We concentrate on these global, or ring-wide, functions because they represent the bulk of the machine's equipment and, consequently, the bulk of the control system's electronics. 
With this prelude we can now discuss the organization of the interface electronics for the type of environment depicted above. The natural and attractive method of organizing the interface electronics at the local control station is to employ an approach of functional modularity that recognizes the decomposition of the machine into its small number of functional subsystems. Figure 2.4 depicts the level of modularity we have in mind.

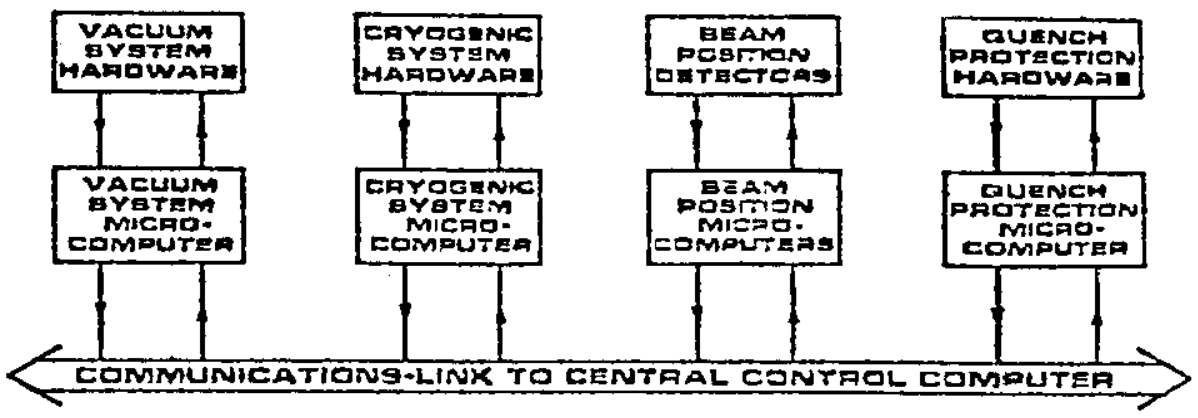

FIGUAE 2.4

FUNCTIONAL GRGANIZATION OF THE LOEAL CONTROL STATION

By exploiting the symmetry of the machine, one subdivides a huge interfacing effort into the simpler problem of how to support the equipment in a single, typical equipment enclosure; by modularizing the interfacing electronics within each equipment enclosure into a (smali) number of microcomputers one achieves an organization of the electronics which bears a natural relationship to the accelerator subsystems being monitored and controlled and which can be configured to meet their individual needs; by employing bus-oriented microsystems one achieves a finer level of modularity - the board level - which makes provision for using a common set of boards for many different microsystems while still leaving open the possibility of upgrading and expanding each microsystem on an individual basis. 
Note that this approach does not require exact symmetry of the equipment in each enclosure. If cryogenic equipment appeared in only every third enclosure, the cryogenic microsystem would, of course, appear in only every third local control station. In addition, major differences in the vacuum system hardware of two different equipment enclosures, for example, can be reflected in configuration tables that are either stored in PROM of the vacuum system microcomputer or downloaded to it from the central control computers. (See Chapter 6). Reasonable differences in the hardware configuration of two different vacuum system microcomputers are relatively easy to manage in this way. Software management, however, is a much more complex and difficult task. A configuration which required a totally different program in each vacuum system microcomputer would be a nightmare to manage. With the cooperation of the machine designers and builders it should be relatively easy to ensure that identical software is used in each of the many vacuum system microcomputers.

The organization illustrated in Figure 2.4 regards the amount of processing power and the performance of the bus as adjustable design parameters: accelerator subsystems with low performance requirements can select an 8-bit processor and the STD bus; those with moderate performance requirements have 6 -bit processors and the Multibus available to them; extremely high performance requirements could chose the VME bus or perhaps Future bus. Although this ability to "mix and match" is (superficially) appealing, its use is not one we would encourage. It is usually wiser to pay a bit more money and employ the same bus structure and the same processor for all the microsystems used to constitute the local control station. The additional expenditure required to achieve this hardware uniformity is invariably recovered by the significant reduction in software costs that accrue from being able to use common code in the collection of microsystems at the local control station. (See Chapter 7 which discusses the concept of "mass produced" software.)

Even as a schematic, Figure 2.4 is incomplete in several respects. It makes no provision for programmable instruments which use the GPIB bus, and it provides no "catch-all" or general purpose interface for signals that are not associated with one of the major machine subsystems, such as an enclosure temperature or humidity sensor, for example. These items usually represent only a small fraction of the total number of signals which need to be interfaced and ordinarily do not require substantial local processing performance; they can usually be accommodated by the addition of a general 
purpose or "catch-all" microsystem to the local control station.

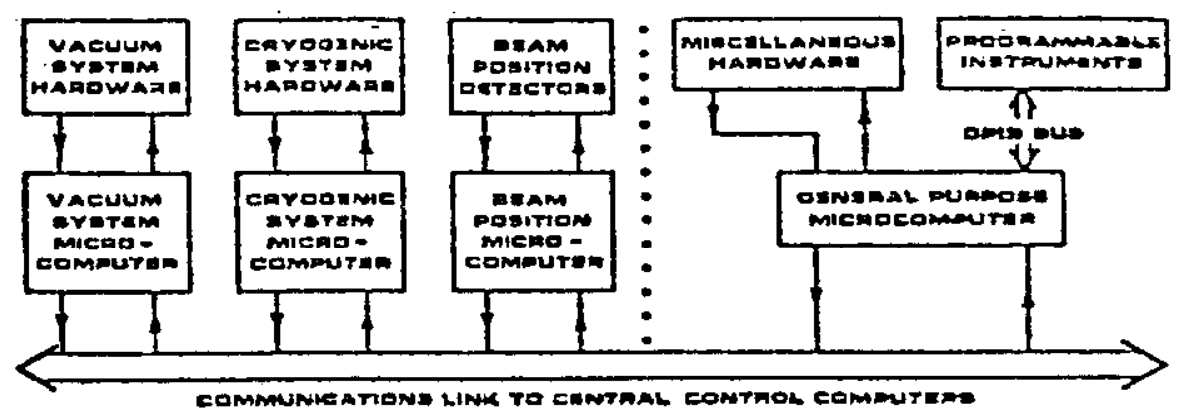

FIGUAE 2.0 ORGANIIATION DM THE LOCAL CONTMOL STATIJN

Even with this addition, the organization of the local control station is still incomplete, since it does not indicate the level, if any, of local interactive facilities for monitoring and controlling the equipment - a topic worthy of an extended discussion.

\subsection{Interactive Facilities at the Local Control Station}

Once a new accelerator has been commissioned and placed into daily operation, the vast majority of all needs for human beings to interact with the control system occur at the central control room. Even so, the system design should make provision for interactive facilities at the local control station. Before the machine can ever function as an accelerator, each of its subsystems and each of their components must be installed, debugged, checked out and placed into operation. Interactive facilities at the local control station are an indispensible tool for debugging and checking out equipment at the earliest possible stage in the construction cycle.

Even when the machine has reached a state of routine operation, there are still equipment failures, maintenance operations and trouble-shooting procedures that require personnel to work periodically at the local control station. 
In these situations the maintenance or diagnostic process can be complicated, error-prone and perhaps even ineffectual without the use of some type of local interactive facility.

The additional hardware needed to support interactive facilities at the local control station need not be elaborate and need not consume much space. A subset of the components used at the control center would be adequate (Cf. Chapter 3): a keyboard, a method for cursor control and a display monitor, for example. In some environments it might be practical or necessary to package these components as a portable diagnostic tool, in the same sense and spirit that an oscilloscope is a portable tool.

The simplest and most desirable way to interface this interactive hardware is by the addition of one more processing subsystem to the electronics at the local control station. It, too, should be a bus-oriented subsystem, using the same bus structure and the same processor as the other microsystems at the local station. With this addition the local control station takes on the organizational structure illustrated schematically in Figure 2.6.

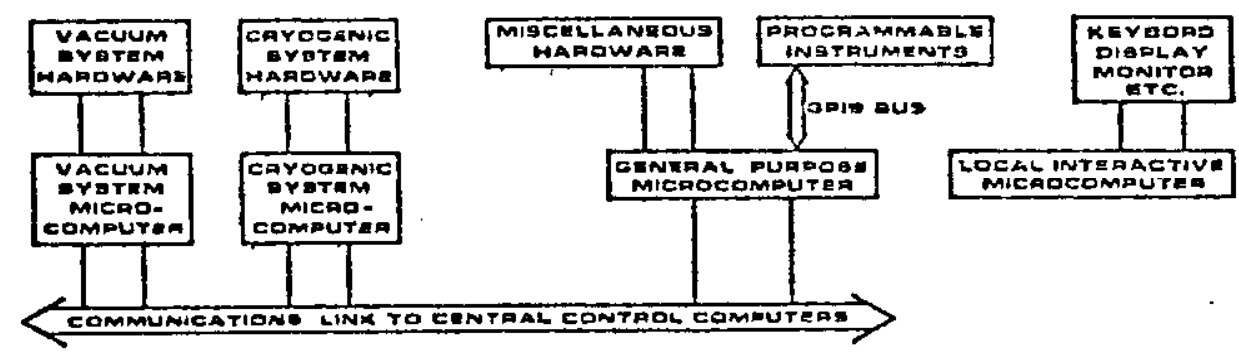

rovar e.s

MARDWAAE OROANUZATION OF TME LOCAL CONTADL ETATION

The communication hardware which links the interactive subsystem to the other microsystems at the local station is not shown in Figure 2.6. This hardware could be a series of point-to-point links but serious consideration needs to be given to the alternative of using one of the network structures discussed in Chapter 4. 
The tools, the methodology, the constraints and the guidelines for developing the software needed by all these microsystems is clearly a topic of major importance. Chapters 5 and 6 discuss the software design, not only of the microsystems, but also of the other processors in the configuration of a large accelerator control system. 
CHAPTER 3

CONTROL ROOM FUNCTIONS

3.: Control Room Functions

3.1 .1 Why have a control room?

Accelerators have not always had control rooms. 19 When the machine was small enough to be accommodated in one hall, power supplies and other devices were often arrayed on the safe side of a shielding wall and controlled directly from there. Even large modern machines often have components which are still controlled in this way. This kind of direct control is simple and foolproof but limited, since knobs and switches on various devices cannot be coupled and procedures involving a sequence of interventions on several units cannot be automated. When the machines became larger, remote control became essential for reasons of geography. once the data and controls had been converted to remotely accessible form, the application of computers was an easier step, whose advantages from the point of view of automatic coupling and sequencing opened a whole new world of possibilities.

Thus the operator controls become peripherals of a computer, which in turn speaks to the machine. Advantages of this arrangement are twofold: first of ali there is some freedon about where the controls can be, so that everything can be in one place and one or two people can control a machine covering a large site. Secondly the operator's console speaks to a computer, not directly to the accelerator, and this computer can act as a translator, allowing the operator interface to be 
friendly, while the computer takes care of the technicalities inherent in the transmission and use of the information.

3.1.2 The flow of information to the control room

Not all information flows to the control room through the computer. Analog signals, TV pictures and some interlock circuits go over other routes. Even in these cases, the ubiquitous computer has its role. Analog signals are usually available in far greater numbers than can be simultaneously shown on oscilloscopes, and here computer assisted switching can be used for a controlled selection process which saves cable and brings order out of chaos. The same is true of TV signals and the screens on which they are displayed. Interlock circuits must indeed be hard-wired, but even here a degree of computer monitoring can make the difference between knowing that the machine has stopped and knowing why.

All other information actually flows through the computer. Here the possibility of data reduction (for incoming information) and data expansion (for control) arises. A human operator has a very limited capacity for originating or absorbing information, and matches rather badly to an elaborate machine. The computer can redress this mismatch.

Data reduction can often be achieved by having the computer check variables to find out if they are as expected or within required limits. Only abnormal conditions are reported, allowing the operator to scan a few exceptional cases, rather than everything. Data expansion is used to allow a single instruction to cause a sequence of things to happen, or to cause elaborate functions to be sent to many locations on the change of a single parameter by the operator.

3.1 .3 Special purpose and general purpose consoles

When, as is generally the case, the accelerator complex consists of several major subsystems, or even several component accelerators, more than one computer may be involved. When each subsystem has its own computer and each computer has its own console, the human interface may be quite different for each subsystem. When the subsystems have been commissioned at different times, and even under different management, this is often the case. 


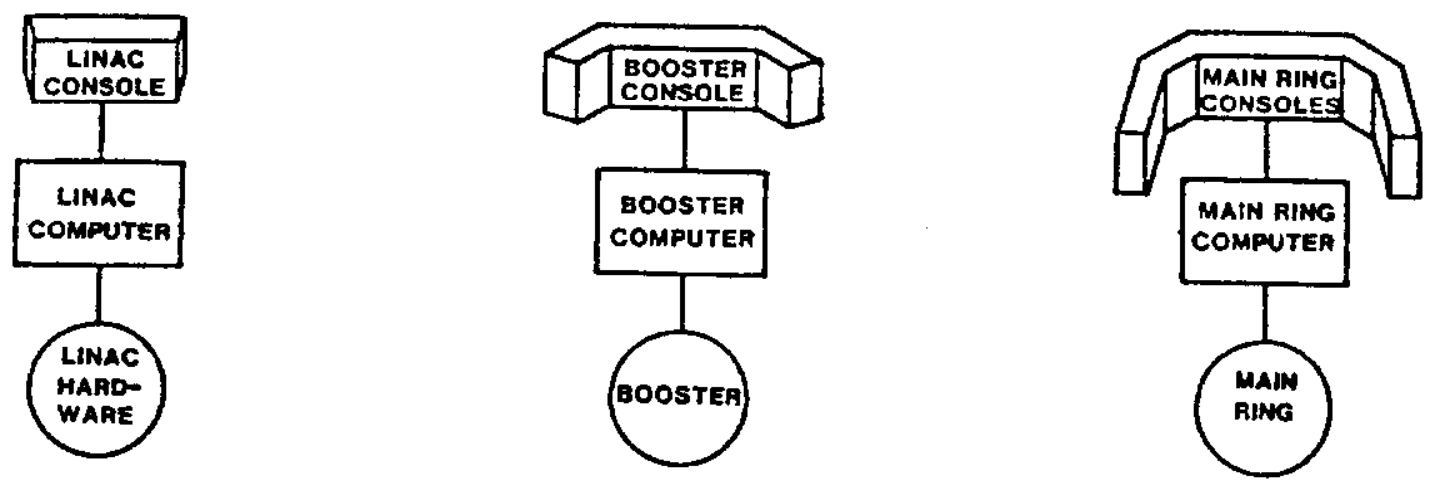

FIGUAE 3.1[A] DEDICATED SYSTEM.

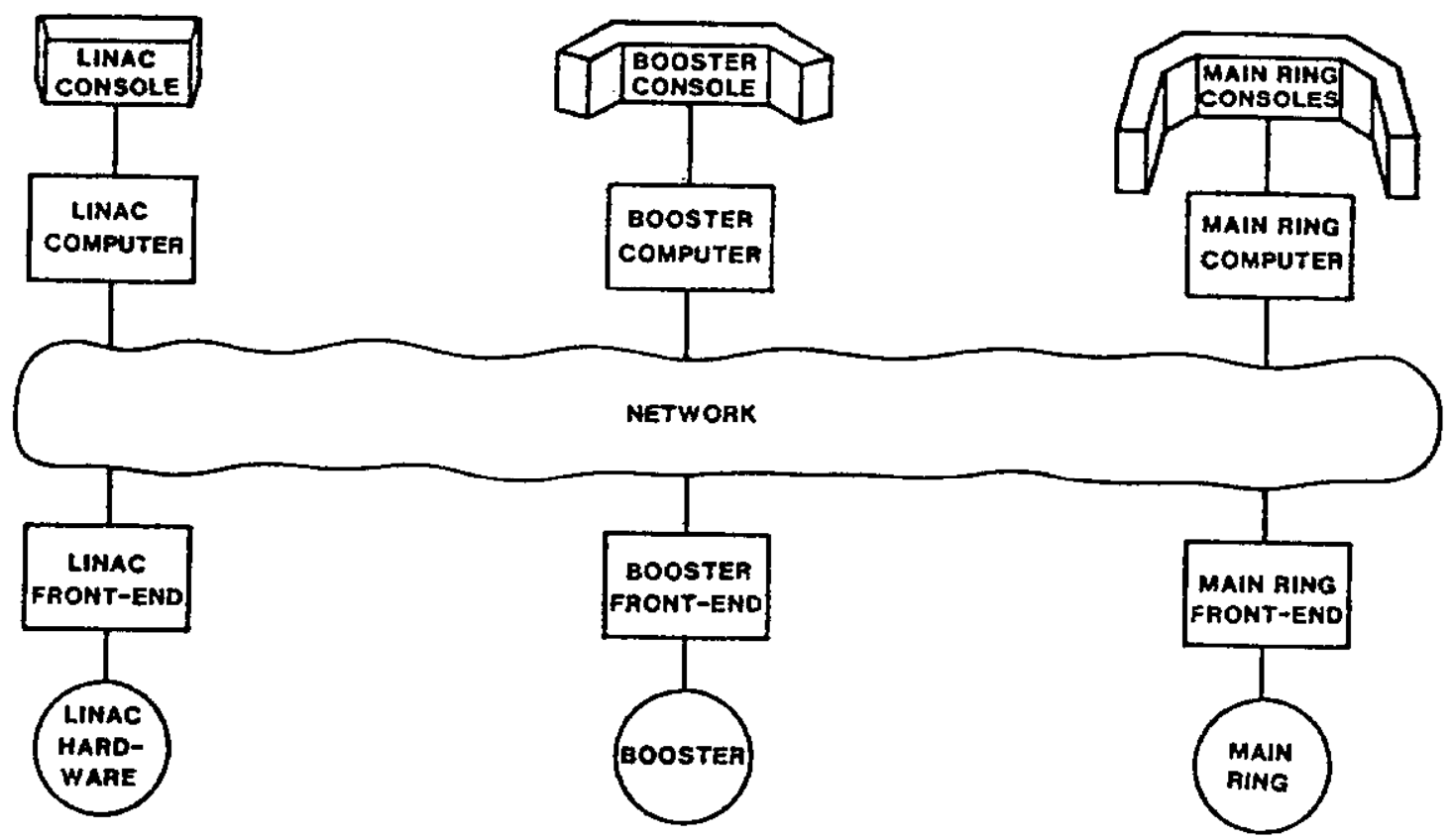

FIGURE 3.7[E] SYSTEM WITH DEDICATED COMPUTERS AND CONSOLES.

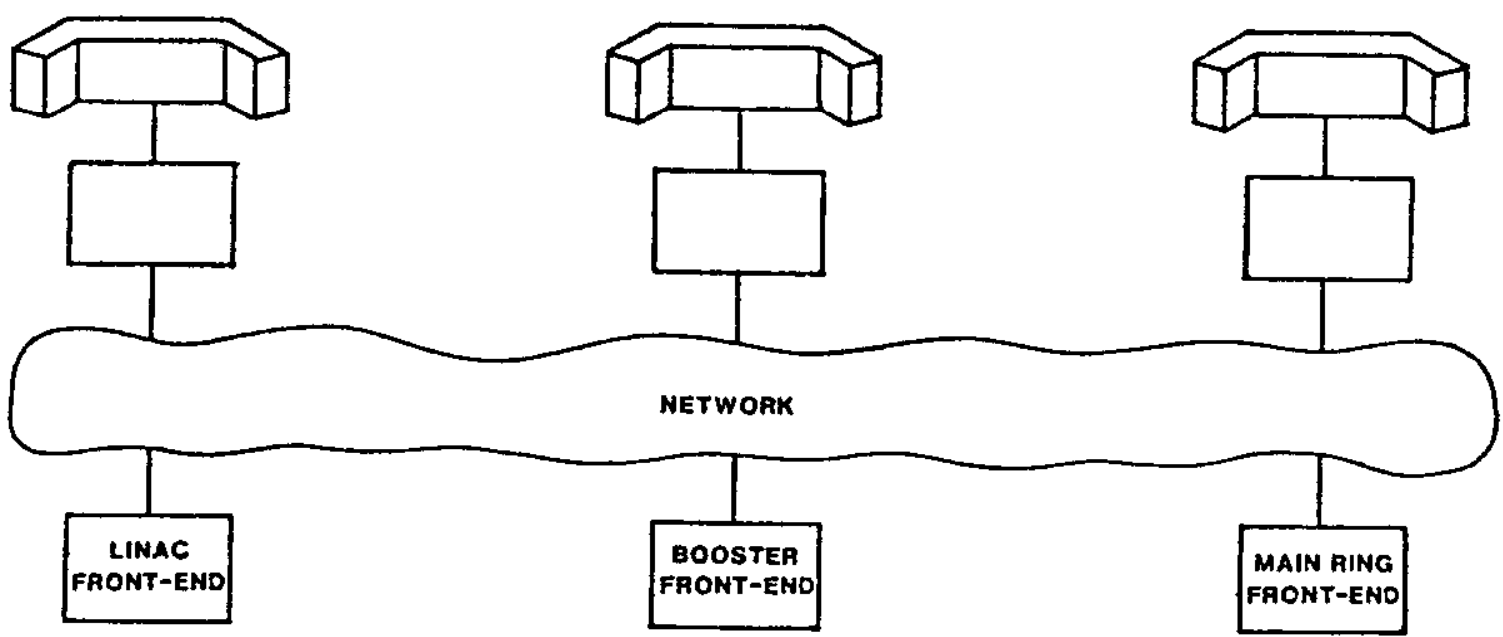

FIGUAE $3.1(C)$

SYSTEM WITH GENERAL.PLAPOSE CONSOLES AND CONSOLE COMPUTERS. 
Two levels of integration are possible. The first is to make a conscious effort to cause all the consoles to behave in the same way. The second, and more extreme, is to connect all the computer systems up in such a way that one console, or a number of identical consoles, can control and view anything. This last stage of integration has been used on some of the largest accelerators, but it has implications in the organization of the teams involved and of the mode of operation. Some people just don't like such global control to be possible, and certainly the provision of general purpose consoles must be accompanied by much thinking about access restrictions and the division of responsibilities between teams.

Even general-purpose consoles are not completely interchangeable. For some analog signals it may be technically too difficult or at the least expensive to display them everywhere. Personnel access to the accelerator itself must be monitored at a unique place for operational reasons. Some consoles may be more elaborately equipped with displays to a level of quality which the budget does not allow everywhere. Still, once the principle of general-purpose consoles has been decided upon, homogeneity should be encouraged at least to the point where there is no need to write different computer programs for different consoles.

\subsubsection{What does a console look like?}

Since everything passes through the computer, the devices mounted on the console have a tendency to be the kinds of devices generally associated with user interfaces on computers. Thus we have a choice of displays, and a variety of buttons, knobs and joysticks.

A number of kinds of displays exist, and all can be found on accelerator consoles. The character by character type of display, using plasma, LED or liquid crystal technology has a tendency to be expensive, but a few seem to appear in most control rooms. They are often used to display some salient parameter, like beam intensity or access status, to which it is important to draw attention, and which requires a conspicuous dedicated display. Most displays used on a modern display desk, however, are of the cathode-ray-tube kind. Here a number of possibilities exist.

The longest-lived CRT device has been the storage display. Storage displays give a pleasant green image of extremely high resolution for a comparatively low price. 
They exhibit a unique feature which is useful in control rooms, namely that images fade with age, enabling information from the most recent cycles to be distinguished visually from older ones. By taking advantage of advancing technology over the years, their major drawbacks, low brightness and short life, have been overcome. Their remaining disadvantage with respect to other kinds of display, the fact that selective erasure is impossible and that therefore moving elements more elaborate than a cursor cannot be portrayed, is not very serious in the control field. On the other hand, as the digital memory used by competing devices gets ever cheaper, the storage display will probably lose its remaining technical and commercial advantages and go out of use completely.

Continuously-refreshed displays can be produced in two fundamentally different ways: either the individual vectors can be drawn with directed strokes of the electron beam, or the beam may perform a systematic raster-scan with selective bright-up to display the information. The former method has the disadvantage that the drawing-time depends on the complexity of the plot and that therefore flicker is unavoidable for elaborate pictures. Raster scans are invariably used nowadays, and the only choice is whether to use a standard TV norm ( 525 lines in the US, 625 in Europe) or to go to higher and non-standard resolutions. The TV standard has the advantage that all components can be purchased in a competitve market, and that therefore monitors, hard-copy devices, multiplexed carrier systems and so on can be obtained cheaply. Its disadvantage is a limited resolution, especially noticeable in color and when displaying characters. In particular the very usua? character format of 40 , 80 -character lines is not possible on a standard color display, unless extra information is packed into the interlace frame, which effectively doubles the resolution but increases jitter and flicker, two irritating features for an image to have. A drawback of the high-definition raster display, on the other hand, is that at present hard-copy may only be obtained by photography.

The picture illustrates the various possibilities which exist with regard to the interlacing process. A picture refreshed 30 times a second will appear to flicker if the successive frames differ significantly in information content. In principle, therefore, one has the choice between an apparently stable image or one containing twice as much information but suffering from flicker. In addition it is to be noted that since a 
point on the interlace frame is drawn $20 \mathrm{~ms}$ after the point directly above it, irregularities of as little as a few nanoseconds in this $20 \mathrm{~ms}$ delay will cause an observable difference in angle between the position of the two dots, resulting in an annoying jitter. Ordinary television pictures suffer from both defects, but they do not normally depict structures which are studied in detail like characters and graphics.
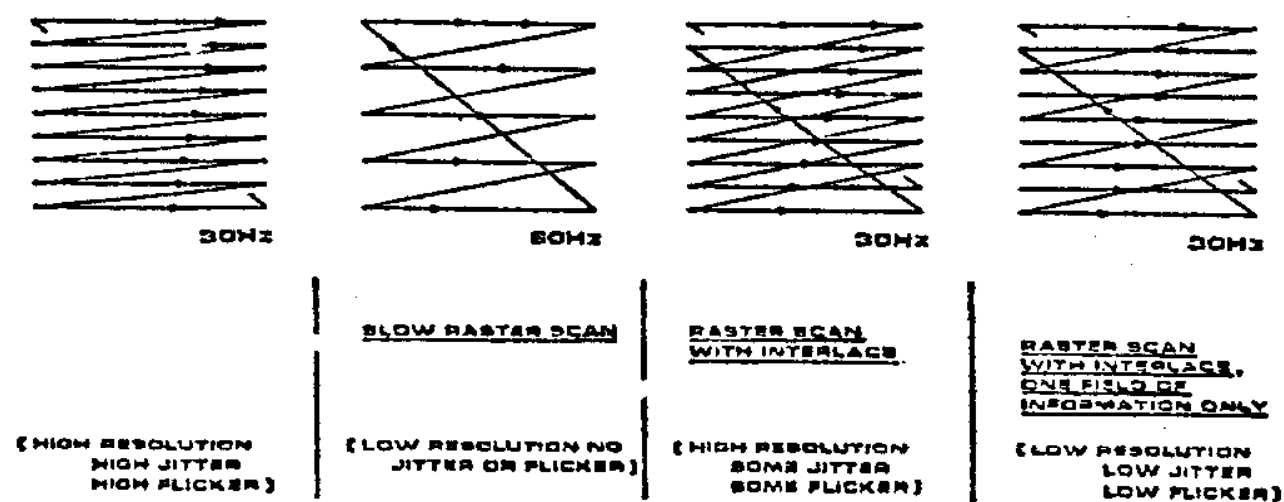

EGUAY S.R AASTEM gCAN TECMNIOUEA

The operator interacts with one of a number of devices. Bistable mechanical switches have a tendency to be absent, as they cannot be reset from the computer. Monostable push-buttons are more usual. Buttons with programmable legends are taking over in more and more control applications. These "touch buttons" operate on a variety of physical principles, but in every case they allow the computer to present the operator with a choice depending on the current state of the machine and its computer system. In addition, touch-buttons, being electronic, are often more reliable than mechanical ones.

Analog control may be passed from an operator to the machine by means of knobs, balls and joysticks. It should be noted here that knob control of variables is far less useful or necessary than it was in the days before computer control. This represents the natural progression of accelerator physics from an art to a science, as well as the growing awareness of the risks of indiscriminate knob-twiddling on an accelerator of high intensity or one in which the stored beam represents a considerable investment. Nevertheless, some, direct knob 
control is done, especially on fast-cycling machines: other installations exclusively use these devices to control setting variables in the computer, and then to use push buttons or touch buttons as validation devices, to send the desired setting variable to the hardware.

The knob, ball or joystick is often used in conjunction with a cursor to inform the computer of an operator selection. In this guise it is the precise equivalent of a touch-button system: either can be used to provide hierarchical menu-choices, and the decision is mainly one of taste. Touch buttons usually allow an attractive and elegant choice from up to about a dozen possibilities. Ball-and-cursor systems allow the menu to be much longer and the text describing the options to be more complete.

The physical design of the control desk and the room in which it resides should be given some thought.20 People will spend long hours using it, and they should be comfortabie. The console, which as we have seen contains half-a-dozen screens, at least one oscilloscope and some knobs, balls and buttons, should also provide a keyboard for computer input, a writing surface, and perhaps even somewhere to stand one's coffee. The table-surface will be about the right height for sitting on, and therefore sitting on it should be possible without causing damage. Attention should also be paid to kneeroom and the ability of people to work in pairs. It is nice to have a free-standing console with the operator able to look over the top, perhaps at extra displays on the front wall of the room. If, however, this restricts the number of displays which can be provided, there will be a tendency for extra devices to be balanced on top of the console. In this case one might as well have a wall-mounted console. It's nice to know that some things are just a matter of taste!

\subsection{Control Programs}

\subsubsection{Who Writes Control Programs?}

However much software is involved in a control system for an accelerator, the only part immediately apparent to the user of the machine is that which controls his interaction at the console. The design of this software is always a compromise. Whilst it is desirable that using the accelerator should be as easy as playing a good video game, there is no point in concealing the fact that an accelerator is an extremely complex research installation, and that no operation 
should be precluded in the interests of simple appearance at the console. The design of a console strategy must therefore take into account the three classes of users of the console, and attempt to satisfy the very different requirements of each.

The console is used by three classes of people. A particular individual may sometimes be a member of different classes on different occasions:

First, there are the members of equipment groups. These are the people who commission the actual subsystems, such as refrigerators, power supplies and vacuum pumps. They may well have tested the material in the field, and be in the act of integrating a number of devices from the control room. In general, such people will have been working with fairly crude computer control interfaces in the field, and will not mind using similar methods in the control room. Typical methods of interaction are by keyboard and character display, and it may even be that there is some advantage to using compatible software in the field and at the control-center.

Secondly, there are the machine physicists who commission the accelerator when the subsystems are in the main debugged and working. Here the emphasis is on ease of operation. The machine physics session is limited, and quick, convenient operation is at a premium. It must be easy to choose and display variables in convenient groups, to put up comprehensible, well thought-out plots and make hard-copy from them and to store the relevant parameters at the touch of a button so that it shall be possible to return to a given operating situation at the end of an experimental sequence. The machine physicist is highly qualified and he knows his machine, so there is no need to talk down to him, but convenience and speed are vital.

Finally there is the shift operator. Programs used by these people often arise from those used by the machine physicists, and are therefore unsuitable for day-to-day operation. Operator programs should allow easy startups or re-start, the recording of post-mortem information for the use of experts later, the study of long-term stability and the improvement of operating conditions. Ideally, these programs should be developed by the people who are going to use them, using the machine physics programs as a basis. In addition to getting the work done by the right people, this technique adds interest to the operating task and helps with training. 


\subsubsection{Interactive Methods}

A whole spectrum of console interaction processes is possible. Control may be exercised at the level of individual vacuum pumps, or comprehensive programs can be supplied to control whole complexes. In practice, both kinds of program should exist: it should be possible to vary the current in each quadrupole, but it should also be possible to correct and display the entire closed orbit in one operation. The vital thing is not to try and do too much in one program. Some programs should be written which allow the display and control of selected individual devices. Others to perform the global operations. All this implies the existence of a large number of fairly simple interaction programs, and this in turn requires a convenient method of moving from one to the other.

Mention has already been made of touch buttons and bali-and-cursor systems for choosing from menus. Whichever of these devices is chosen, it must be easy to return to the fundamental index and re-choose. The methods used at the Fermilab and at the SPS differ in a sufficiently fundamental fashion for it to be worthwhile describing both here:

At the SPS, a hierarchy of choices is made, of major system, subsystem and so on, on a panel of at most sixteen touch buttons. On arrival at a program, the touch buttons are available as a resource for the writer of the program, for choices inside it. In addition, there are two fixed buttons, to return to the point at which the previous choice was made, and to return to the "trunk" of the tree.

At the Fermilab Booster and Main Ring the choice of program is made from a list with a ball and cursor. The hierarchy has one level only. Since the choice of programs is greater than can be displayed on one page, a "next page" choice is available. A selected choice can either be of a group of variables to be displayed and controlled, or it can be a free-form control program using knobs and other console devices. The fundamental choice of which accelerator is to be controlled from this console is not a console choice.

The new Tevatron system at Fermilab allows either arrangement, so in due course it will be possible to compare the two. 


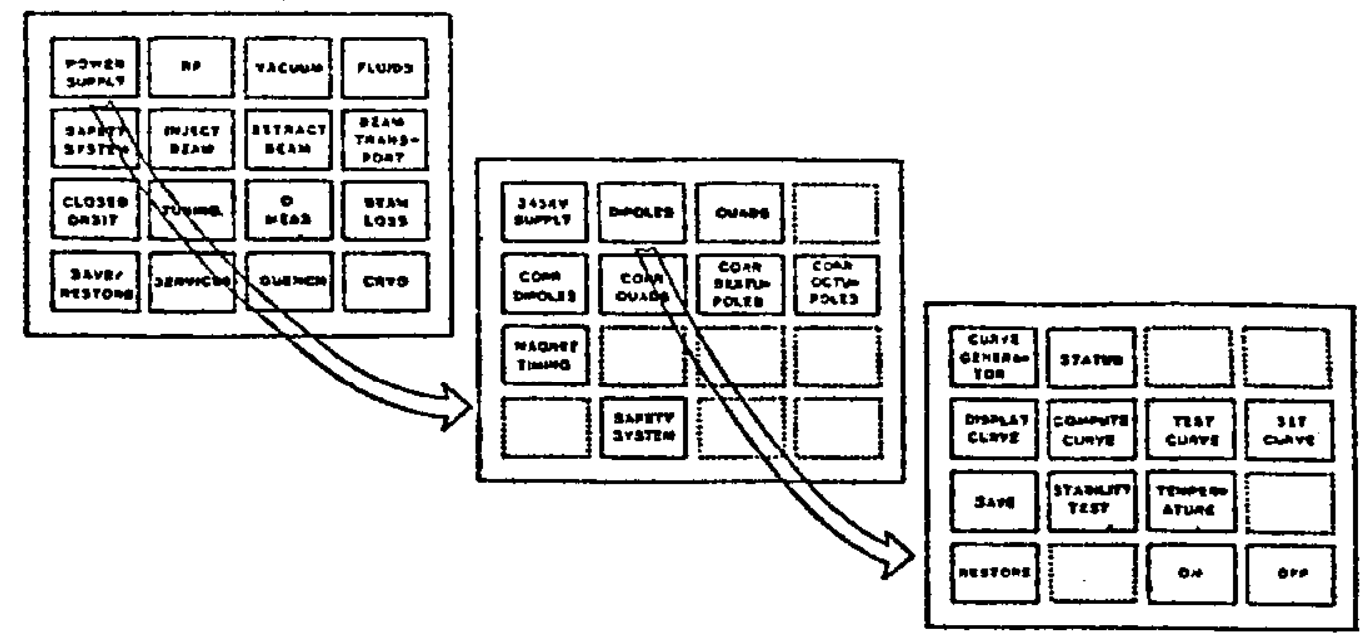

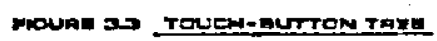

The setting of variables usually requires the display of the current or other reading, continually refreshed, a setting value, and perhaps some parameters for operator guidance on the desired value or limits. Colors can be useful here, to label variables in the course of chenge, variables which are out of range, or requests which the computer is unable to satisfy. Read-back of a whole array of functionally related. measurements is often better done by the display of $a$ graph.

When graphs require more detail than the display technology provides, it may be advantageous to provide "zooming" so that a global picture is supplemented by an enlargement of a selected area of it. Selection of the area to be enlarged can be made by a ball-and-cursor operation on the global picture. Together with the taking of position readings from the ball, this technique can be used to read and set values to very high precision without typing in or reading off values with a number of digits. 
3.3 Control Room Information Strategies

3.3.1 What to display

It is possible to keep an eye on a large and intricate accelerator complex by glancing at $a$ few displays carefully chosen to show the intensity and energy of the beam or beams being used by the experimenters. Since this is essentially a quality control check on the product being exported by the factory, such a restricted display will at least give warning of a drift which affects output. This kind of display, giving magnet ramp voltage and beam current on an accelerator or intensity and predicted lifetime in a storage ring, represents a minimum of permanent information to tell the operator that all is well. It is often called a "dedicated display".

A dedicated display is far from sufficient. The machine may be working well, but drifting to disaster. Such a situation can be avoided by continually checking major subsystems by means of scheduled computer programs and putting up further comfort displays for the subsystems. Infortunately, such a policy soon uses up two very precious resources: bandwidth and display space. Since all information passes through the computer system and its associated network, if any, bandwidth may not be in unlimited supply. As far as display space is concerned, an accelerator has enough subsystems to ensure that a full display would be impracticable, even if it were easy. The mental strain of continually scanning a large and elaborate panel is such that the risk of missing something important is quite great. Fully refreshed displays should indeed be available on demand, so that when a particular subsystem is under critical study its parameters can be watched. In normal operation, however, the parameters should be watched by computer program and only abnormal conditions reported.

This tendency to reduce the amount of unsolicited information display provided has followed the introduction of computers for control. Computers render it both more difficult and less necessary to provide dedicated displays of everything. This trend is being noticed even in very conservative fields like power station control rooms and the cockpits of large commercial jets. 21

There should therefore exist an alarm screen for reporting exceptions. Since it is the only window available for looking at those parts of the system not 
specifically being studied, it is desirable that the alarm screen be dedicated to that purpose and that suppressing the reporting of alarms should be difficult to do inadvertently. Alarms need not be very specific, since in principle the operator has enough facilities to investigate more deeply once he knows there is something wrong. Techniques for scanning and displaying alams will be discussed below.

\subsubsection{Alarm Scanning and the Display of Alarms}

Alarm scanning is usually done by a program loop which reads each status item or analog measurement, compares it with the desired condition, and reports abnormalities up the control hierarchy towards the alarm display. This simple description conceals a number of problems:

1. The program acquiring the hardware variables may have to compete with central control for access to the hardware.

2. Reading the hardware requires access to a data base specifying the hardware address of each item, as well as its scaling factor, units and other parameters.

3. Deciding whether the acquisition is correct or within limits requires access to additional data base items specifying the desired state of each item.

4. Repeated reporting of an undesirable condition, every time a scan is done, can be a nuisance. A well-constructed alarm system should report only changes, and in addition does not report at $a 11$ a condition which is obvious in view of the existence of some other alarm.

All this means that alarm scanning programs should have access to the same kind of information which is available at the console: they should be aware of the operator's requested changes, the presence of relevant alarm conditions elsewhere in the system, and the latest alterations in the hardware layout. This is a pity, since it gives rise to a conflict: bandwidth requirements dictate alarm scanning in a position near to the equipment (such as a microprocessor in the same crate as a subsystem). The operational requirements given above point to an omniscient alarm scan, having access to the system data bases and the operator's knowledge. This 
paradox cannot be fully resolved, and a practical alarm system is a compromise consisting of rather simple-minded local scans reporting to a central system which filters the results for screen presentation.

The fact that lack of an alarm message will be taken by the operator as meaning that there is nothing wrong with the system means that an alarm system must have the operator's confidence. The central alarm program must be programmed to expect an "I am alive" message from each subsystem at regular intervals, and to put up an alarm of its own if any subsystem fails to report.

\subsubsection{Refreshed Displays}

As mentioned above, refreshed displays keep the control computers and their associated communications systems very busy. Nevertheless, refreshed displays are an important feature of computer control systems, and they must be supplied, when required, at a minimum cost in resources.

First of all, the information displayed in this manner should only appear on request. This means that the page or graph should appear on a general-purpose screen when needed and with a refresh rate which is meaningful with respect to the requirement. Some system designers allow the scheduled program supplying such information to time out or abort after a certain period unless continuance is specifically requested. No variable needs to be scanned for refresh purposes above persistence-of-vision speeds; few more than once per second.

Secondly, it should be made easy for the operator to ask for the presentation of fewer variables at a time. This is achieved by allowing him to configure pages with just those variables that are interesting, or by arranging for the preparation of global displays with little detail, with easy switching to local displays of selected areas with full detail.

Finally, there are tricks for reducing the information continually refreshed on the display. Information can be compressed for transmission, as when there is no change for a sequence of readings, or certain parts of the display can be designed for more frequent display than the rest. Such tricks can introduce considerable complexity, which is undesirable, and should be avoided except when actually trying to relieve information overloads. 


\subsubsection{Analog Information}

However digital and computer-oriented control systems become, the oscilloscope is a very important diagnostic tool, and many important facets of accelerator study require the presentation of repetitive time-related waveforms. Cables drawn from instruments directly to oscilloscopes in the control room are easy to use and easy to understand. Unfortunately a few signals of this kind will incur more cost in cabling, display space and operator attention than a computerized control system dealing with thousands of variables. In addition, the very large physical dimensions of modern accelerators mean that the noise-free transmission of analog signals with compensated control of attenuation over a useful frequency range can be expensive, if sufficient precautions are taken to ensure that the signals are meaningful when they arrive in the control center.

Various remedies are available. Only $z$ few well-compensated cables are supplied from remote buildings, and selected signals are routed via these cables to a few oscilloscope traces. Selection of the remote signals and their attribution to individual display facilities is performed by a kind of telephone exchange remotely controlled from a console via the computer control system. It is not cheap or simple to do this well, but both the major accelerator control systems at CERN have comprehensive analog signal selectors of this kind. At the Fermilab the more modest approach has been used of displaying a strict minimum of such signals, and hard-wiring those.

All the systems described above deal with signals from D.C. to $1 \mathrm{MHz}$ or so. Higher frequencies can be dealt with by laying specialized (and very expensive) low-loss coaxial cables and by ensuring at design time that the distance from the signal source to the control room is small. A number of high-speed sample digitize and store systems are available commercially, which allow the signal to be frozen in a digital memory and then read out digitally or in some analog form. If the digital form is used, an array of numbers can be transported to the control room and plotted there at regular intervals with great faithfulness to the original signal, and effective bandwidths in excess of one gigaherz are obtainable by this means. Apart from the very high price per signal, a drawback of devices of this kind is that the controls for synchronization, sensitivity, time-base frequency and so on have to be manipulated remotely, and all this has to be interfaced and programmed. Still, 
they do provide a means of displaying the RF stmeture of the beam remotely and with confidence, and so are part of the amory of the machine physicist.

This kind of device can also be used to read a sampled waveform into the computer for subsequent computational analysis, though if the frequency is lower it might be possible to use a cable to transport the signal to the control room. This costs a cable but saves remote control of the oscilloscope controls. 
CHAPTER 4

INTERCONNECTION OF THE LOCAL AND CENTRAL STATIONS

\subsection{Introduction}

Chapter 2 presented an organization of a set of geographically distributed local control stations, each of which contained a number of processor-based subsystems. Chapter 3 described the organization of the central control station which used a collection of computers over which different applications, requirements and hardware resources are distributed. In this chapter we discuss the methods and technology for connecting these elements together to form an integrated system.

It is important to note that the elements being interconnected are processors; we are concerned with the methods for connecting microcomputers to each other, microcomputers to minicomputers, and minicomputers to each other. Consequently, to a large extent this chapter will represent a distilled summary and a short status report of the technology and techniques for interprocessor communication, i. e., for computer communication networks. We make every attempt to minimize the usage of arcane communications terminology and we limit ourselves to those communications concepts which, potentially, have a direct application to accelerator control systems. 
The networks that most people have heard about are ARPAnet, TELEnet and TYMEnet, all of which extend over thousands of miles. Such large-scale networks and the technology they use are not particularly relevant to accelerator control systems, which are typically concerned with communications systems that extend over distances of 40 miles or less. Networks of this type, $i . e .$, those that are geographically confined to a small number of miles are known as local area networks.

A tremendous amount of effort on the design, development and standardization of local area networks is currently being expended by both the data processing industry and the communications industry. The motivation for this extreme interest in local area networks is that they appear to have applications that are attractive in quite a large number of different environments. Local area networks can be used for efficiently sharing the computing resources on a university campus in a cost-effective manner; they can provide the structure that is necessary for automating complex processes in both light and heavy industries; they are a key ingredient in managing the flow of information in the modern office environment, and so on. It has been projected that the sales of equipment for local networks will grow from $\$ 100$ million in 1981 to over $\$ 5$ billion in 1994.22 A typical conception of the modern office environment is illustrated in Figure 4.4 .

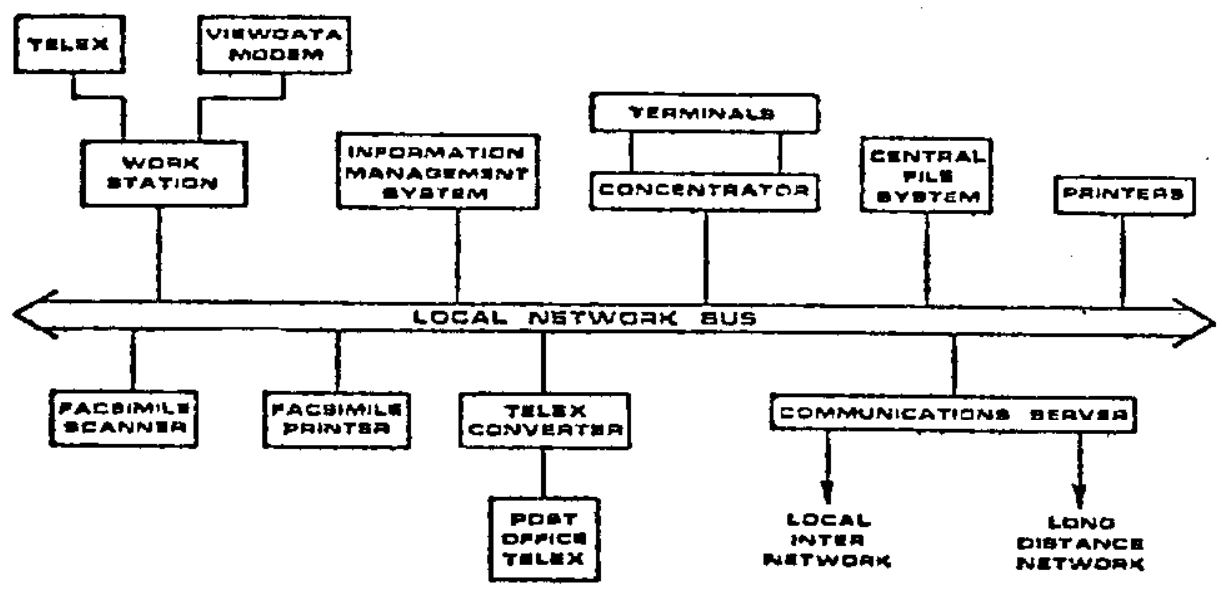


The phrase "local area network" is not a precisely
defined term. Rather it is used to characterize a communications system, usually owned by a single organization, that covers a limited geographical area (from several hundred feet to several miles), that uses an inexpensive communications medium, that supports high data rates (from 100 kilobits per second to 100 megabits per second, or higher), that is highly reliable, and that can be expanded by the addition of new stations in a simple and straightforward way without disturbing the rest of the network.

There are three primary properties which distinguish one local network from another: the topology, the access methodology, and the encoding scheme.

\subsection{Network Topologies}

One of the important characteristics of a computer communications network is its topology, $i$. e., the physical geometry of the processing nodes and interconnecting links. The topology of a network contributes to and in many cases determines some of the critical parameters associated with the transfer of information from one processor to another: the throughput or number of bits per second that can be transferred from a source processor to a destination processor; the delay or amount of time which elapses until the destination processor receives the information in useful form; the routing strategy or the complexity of the total path over which the data must travel between its source and destination. The most common network topologies are the bus, the star, the ring and the tree.

\subsubsection{The Bus}

A schematic representation of $a$ bus is shown in Figure 4.2 .

The bus or multi-access channel has the property of being fully-connected: any node can communicate directly with any other node without first passing through intermediate nodes. In this topology no path selection is required: every node sees every message but each node is required to select and respond only to those messages which are addressed to it. It is possible to do multicast operations - where the same message is sent to 


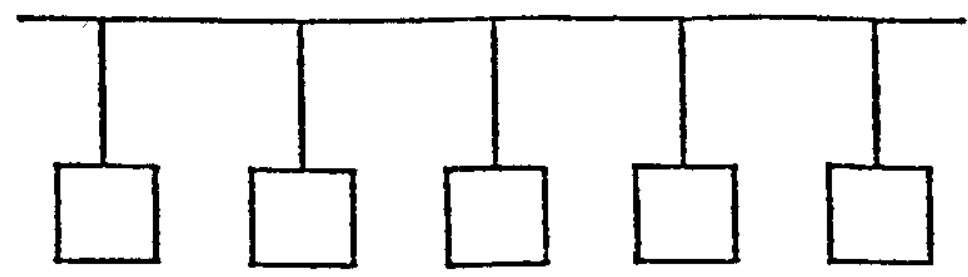

FIGUAE 4.2 THE BUS TRPDLDEY

more than one destination - and broadcast operations where the same message is sent to all nodes on the link. If a node should fail, it is required to present a very high impedance to the bus in the failed state - thereby removing itself from the network. Consequently, should one or more nodes fail, the network continues to function - a characteristic termed "graceful degradation". The bus topology is often implemented, physically, as a passive coaxial cable over which information is transmitted in bit-serial form.

\subsubsection{The Ring}

The ring topology, shown in Figure 4.3 , has many of the same characteristics as the bus.

In a ring network no path selection is required, both multicast and broadcast operations are possible and the transmission medium is commonly implemented as either coaxial or fiber optic cable. Unlike the bus topology, however, each node of a ring network ordinarily connects to the transmission medium with an active repeater. Most ring networks are bit-serial systems in which messages circulate in one direction on the ring, usually being amplified and repeated at each node, until they are removed by the original transmitter. A node can acknowledge a message by setting flags in the message as it goes by.

A ring network appears to be quite vulnerable from a reliability perspective; a fault occurring at a single node could result in disabling all communications. To deal with this problem, the first repeater downstream 


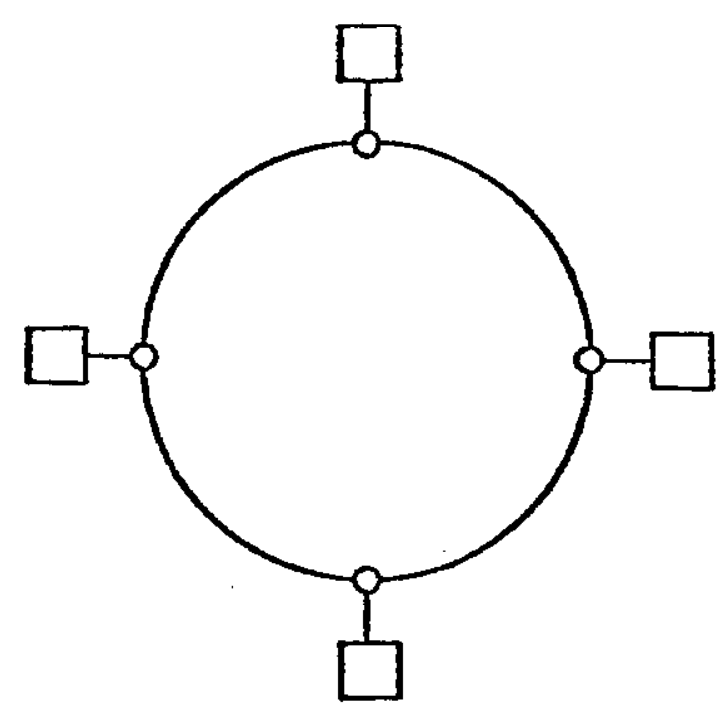

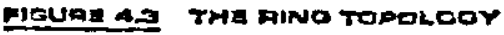

from the fault is usually required to automaticaliy detect the fault and to transmit report information to a designated monitor node. In addition, the repeaters are required to contain bypass relays that automatically open the connection to a faulty node but maintain the continuity of the ring connection through a shunting link. With a good design of the repeaters, a ring network can operate reliably and efficiently in a high availability environment.

\section{$4 \cdot 2.3$ The Star}

The star topology relies upon a central node to provide communications between the other stations and is illustrated in Figure 4.4 .

The star network is probably the most established network topology and was the one chosen for the SPS, where each central node of a tree of stars (see below) operates as a message-routing system. By equipping the processor at the central node with a large number of direct-memory-access channels, it is possible to have a large number of messages in transit at eny instant. 


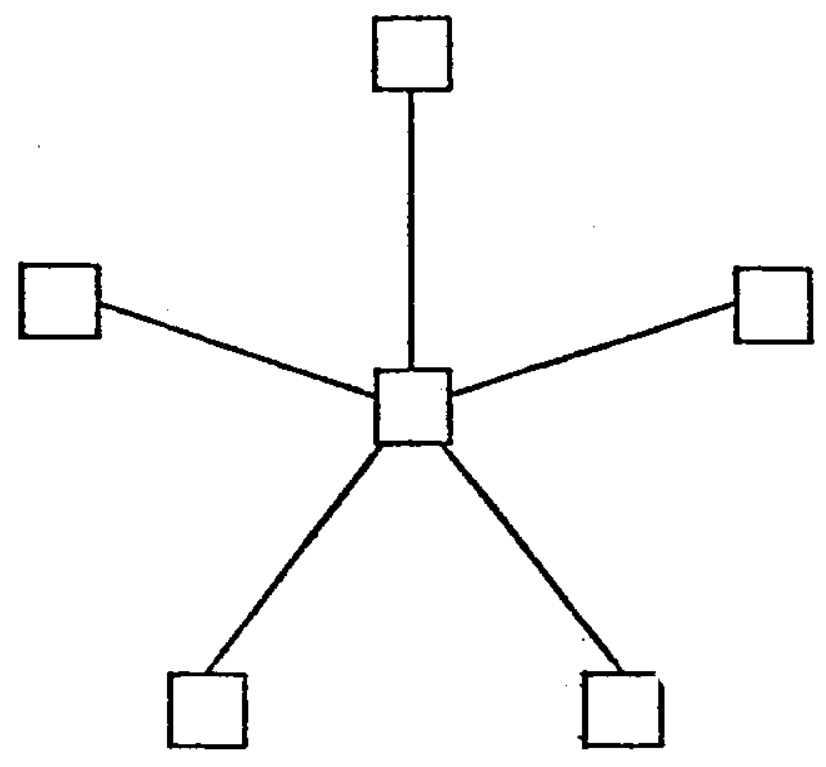

FIGURE 4,4 THE STAR TOPOLOGY

The star network has two major disadvantages. Since all messages must pass through the central node, a failure at this point could be catastrophic. To provide the high level of reliability that is required at the central node it is usually necessary to employ one or more redundant processors at this location in the topology. The presence of multiple processors, however, makes the configuration (and reconfiguration, in the case of a failure) of the links to the other nodes somewhat complicated. In addition, since all messages must pass through a single node, there are obvious throughput limitations to a star network. Because the star network ordinarily has considerably lower throughput than other topologies and because the central node of a star network is so vulnerable to saturation and a potential maintenance headache, star networks are currently unpopular for high-data-rate applications.

\subsubsection{The Tree}

A schematic illustration of a tree network is shown in Figure 4.5 . 


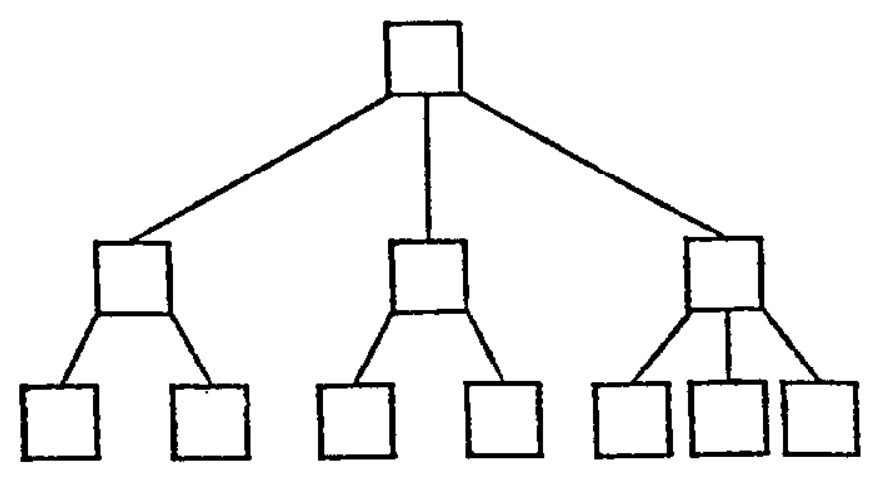

FIGUPE 4.8 THE TAED TOPQLOEY

A tree network is sometimes called an hierarchical network - implying a vertical distribution of tasks, responsibilities or resources among several, functionally distinct, vertical levels. It is easy to build the various levels of a tree network out of stars, rings and busses.

All modern development work on local area networks relies upon either the bus topology or the ring topology. The star and tree topologies are usually reserved for connecting one or more local networks to each other - an aspect of networking which is still in its infancy.

\subsection{Access Methodologies}

In a network configuration of the bus or ring topology some procedure is necessary for managing the use of the communications medium - an access methodology. Early network implementations had a tendency either to divide the usage of the medium into "time-slots" and to allocate each node a number of dedicated slots during which it had complete control of the medium; or to single out one node as a "master" which controlled the use of the medium, typically by polling all the other nodes in the network.

Neither of these access strategies is attractive for a local area network. Both of these procedures are inefficient because they attempt to use only a fraction of the available physical bandwidth. All modern local area networks attempt to make maximum use of the physical bandwidth by distributing the control of the medium over the entire network. 


\title{
4.3.1 Carrier-Sense Multiple-Access
}

\begin{abstract}
A multiple-access methodology allows each node to access the network independently, doing its own housekeeping to ensure that its message gets through without damaging transactions between any other nodes on the network.
\end{abstract}

Let us first illustrate the idea behind the multiple-access methodology by considering the simple bus network of Figure 4.6, which consists of three nodes A, B and $C$.

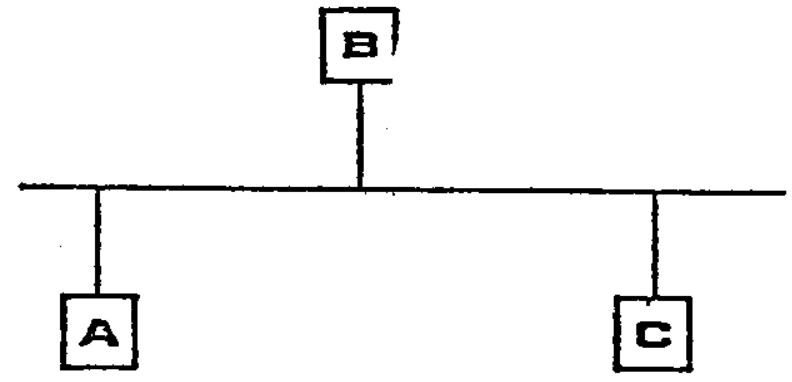

Frougr 4.8

A EIMPLE, THAEE NOOE, घLS NETWOAK

The multiple-access technique allows each node on the network to manage its use of the communications medium independently: there is no master node which controls access to the bus; each node has the same democratic rights to the bus as any other node. However, if node $A$ wants to send some data to node C, for example, node $A$ is first required to listen, or sense, to see if anyone else is currently using the bus. If not, node $A$ can begin transmitting his message; if the bus is in use, node $A$ is required to defer to the current user, to continue sensing until the bus becomes quiescent and to wait a short interval before beginning to transmit. This set of rules for access to the bus is known as the carrier-sense multiple-access (CSMA) discipline. Even with these "rules of the road", however, conflicts between stations still arise.

Consider the situation in which node A has started the transmission of an $\mathbb{N}$-bit message to node $C$, and let 
$t_{d}$ be the length of time it takes for a bit to propagate from node $A$ to node $C$. If neither node $B$ nor node $C$ try to transmit data during this time interval $t_{d}$, then the bus will be managed in an orderly fashion, no conflicts will arise, and the transmission from $A$ to $C$ will ordinarily be successful. On the other hand, if node $C$ starts transmitting during the time interval $t_{\text {a }} a$ conflict for the use of the bus arises, and a collision between the two messages is said to occur. This collision scrambles the data of both messages, so that neither of them is transmitted successfully. The receiving stations can recognize this rather easily, using error-detection and message-consistency logic, but this, of itself, is not enough. The transmitting stations also have to be informed that a collision has occurred, so that each of them can try again.

The standard mechanism by which the transmitting stations learn that a collision has occurred is by requiring the transmitters to monitor and notice the interference on the channel, while they are still transmitting. This mechanism, or algorithm, only works if the propagation time between the two transmitters is less than one-half the time required to transmit the shortest possible message. Suppose, for example, that nodes $A$ and $C$ of Figure 4.6 are the two extreme nodes on the bus, that information is transmitted at a rate of 10 megabits/second ( 1 bit-time $=0.1$ microsec.), and that the minimum message length is 64 bytes $=512$ bits long. Then, the requirement is:

$$
\begin{aligned}
\text { Propagation time } & <1 / 2(512)(0.1 \mathrm{microsec}) \\
& =25.6 \mathrm{microsec} .
\end{aligned}
$$

In this example, the "electrical-length" of the bus must be less than 25.6 microseconds. Relation 4.1 also indicates that one can trade-off bandwidth, or bits/second, with propagation delay or length. By lowering the bit rate by a factor of ten, one can make the "electrical-length" ten times larger.

By requiring that receivers discard colliding messages, and by allowing transmitters to detect 
collisions by restricting the parameters of the bus, the CSMA method can handle the case of a single collision. However, one still isn't "out of the woods". The very occurrence of a collision implies that the two transmitting nodes have, to some extent, become synchronized. If each of them detects the collision and attempts a retransmission right away, a second collision will obviously occur. Clearly, one has to de-synchronize the transmitters.

The standard method of accomplishing this is to require each transmitter, before attempting its $n$-th retransmission, to generate, independently, a randomized delay function so that each station waits a different, randomly determined amount of time, instead of beginning to transmit immediately. This avoids causing a collision each time two or more stations have something to send, and the delays are made progressively longer as the channel becomes busier. This rather complicated access procedure is called the carrier-sense multiple-access with collision detection (CSMA/CD) discipline.

The most important and most highly developed local area network which employs the CSMA/CD access mechanism is Ethernet, a joint development of Digital Equipment Corporation, Intel Corporation and Xerox Corporation. 23 Its primary characteristics are:

$\therefore$ Data rate $: 0 \mathrm{Mbits} / \mathrm{sec}$.

2. Maximum station separation : 2.5 kilometers

3. Maximum number of stations : $\$ 024$

4. Maximum message size : 1518 bytes

5. Minimum message size : 64 bytes

Many studies and measurements have been made on
Ethernet configurations. One of the most detailed
studies was on a network which spanned 550 meters,
connected over 120 stations, and experienced a traffic of 
almost 300 milion bytes per 24-hour day. 24 The studies on this network indicated that 99.18 percent of the transmissions were able to access the channel with zero waiting time. Only 0.79 percent had to defer because another station had control of the channel and less than 0.3 percent collided.

Although the performance measurements on Ethernet networks are quite impressive, the access methodology used by these networks is not suitable for some environments. Since all stations have an equal probability for accessing the channel, the method does not allow giving one station priority over the others. In addition, an occasional message could be delayed for an indeterminable length of time. Because of the possibility of repeated collisions, there can be no guarantee on the maximum length of time a station has to wait before gaining access to the channel. In the office-automation environment an occasional delay of several seconds in the transmission of a message is ordinarily not significant. However, some process control applications have a requirement on the maximum latency that can be tolerated in transmitting commands or parameters from one node to another. If the requirement on maximum latency is a severe one, then an Ethernet configuration could be quite unsuitable for such an environment.

\subsubsection{Token-Passing}

Another popular methodology for managing the access to a channel is a token-passing procedure, which allows each node autonomy when it is its turn to transmit, one node at a time being granted a turn. A token is an authorization for permission to transmit which is passed between stations; possession of the token indicates which station is in control of the transmission medium. Every message transmitted over the channel contains a field which is allocated for use by the token.

A token-ring is based upon a physical ring. Each node examines every message on the ring and accepts those messages which are addressed to it. When a node has data to transmit, it examines the token field to determine whether or not it has permission to use the channel. If the token field indicates that it does not have permission to transmit, the node regenerates the message 
including the token field, without modification, and dispatches it to the next station on the ring. If the token field indicates that a node with data has permission to transmit, then the node modifies the token field as it passes to indicate that the channel is in use, and inserts its data after the token field.

In a ring configuration which supports either broadcast or multicast operation, special action has to be taken to ensure that a circulating message does not repeat from node to node, indefinitely. After the message has made one circuit around the ring and is returned to the originating node, it is deleted and the originating node modifies the token field to indicate that the channel is no longer busy.

There are several advantages that the token-ring offers over the CSMA/CD access method. Firstly, the use of the token authorizes only one node at a time to put a message onto the channel. Thus, there is no possibility of a collision. Secondly, the maximum length of time that any node has to wait for access to the channel is deterministic. If all the nodes have messages to transmit, the maximum wait or latency time is the sum of all the token-passing times and message times. If only one node has a message to send, the maximum latency is merely the sum of the token-passing times. Finally, the token-ring method can be operated on a nonpriority basis or on a multiple priority basis. For example, one node could be singled out as a high-priority station and be authorized for transmission every time it receives the token; another node could be classified as a low-priority station and only be authorized to transmit every second time it receives the token.

\footnotetext{
In normal operation a token-passing procedure is quite straightforward. However, the recovery from abnormal conditions and the initialization of the network can be quite complex. The presence of multiple tokens or the loss of the token, for example, are difficult problems to resolve.
}

Note that the token-passing procedure can be implemented as a logical ring on a physical bus, as illustrated in Figure 4.7 . 


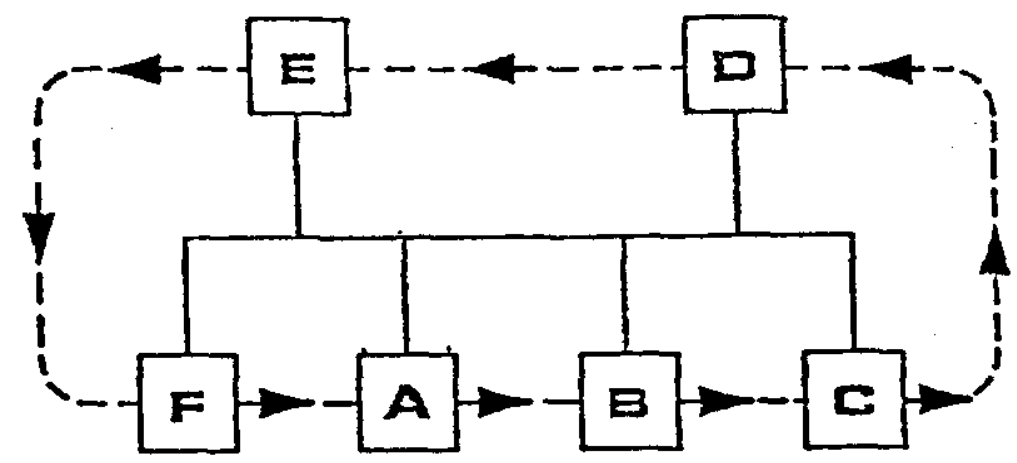

FIGUaR 4.7 A LOGICAL PING ON A PHYgICAL BUS

Logically, each node passes the token to the next sequential node in its logical chain. Physically, it broadcasts the token, with a particular destination address, to all nodes on the bus.

\subsection{Encoding Schemes}

\section{4.! Baseband}

A baseband system is defined to be a system in which information is directly encoded and impressed on to the transmission medium; at any point on the mediur, only one information signal at a time can be present without disruption. Baseband encoding is the simplest, least expensive and most common encoding scheme used in communications networks. It is easy to implement a baseband system with either coaxial or fiber optic cable, requires only a minimum amount of planning to do a robust implementation and is inexpensive to maintain. The major disadvantage to a baseband system is the absence of multiple channels; all information must be transmitted over a single channel.

\subsubsection{Broadband}

A broadband system is defined to be a system where information is encoded, modulated onto a carrier, and band-pass filtered or otherwise constrained to occupy only a limited frequency spectrum on the transmission 
medium; many information signals can be present on the medium at the same time without disruption provided that they all occupy non-overlapping frequency regions. Because broadband systems can support multiple channels simultaneously, they can handle tremendous amounts of information and they offer a high degree of flexibility to the system designer.

In a broadband system one range of frequencies can be allocated to a set of video channeis; another range can be dedicated to the transmission of digitized voice conversations; document transfer, electronic mail and teleconferencing can be supported on a third frequency band; a number of independent Ethernet channels or token-passing channels could be assigned to a fourth frequency interval. Broadband systems can support all these diverse applications simultaneously because they transmit at very high frequencies (up to $300-400$ Megahertz), thereby offering bandwidths of several hundred megabits per second.

All commercially available broadband systems employ coaxial cable as the transmission medium and are proprietary systems. In addition, broadband systems are more expensive per node, require considerably more installation planning and are more expensive to maintain than baseband systems (See Chapter 7 ).

\subsection{Standards For Local Area Networks}

The number of choices that are available to the system designer for implementing local area networks is quite large. In 1980 the IEEE established Standards Project 802 with the express purpose of standardizing, as much as possible, the methods for interconnecting digital computing equipment and peripherals in a local environment. The driving force behind the project's work was the belief that a local area network standard would benefit both equipment manufacturers, by allowing them to mass produce their wares, and equipment users, by allowing them to make purchases at lower prices.

Many people expected Project 802 to endorse an Ethernet-type scheme as the local area network standard. However, in 1982 the IEEE 802 Committee significantly changed its objectives. Rather than attempt to develop a monolithic, universal standard that would encompass all possible local 
area network technologies and applications, it has instead recognized that different approaches might best suit different applications. The most recent draft of the proposed standard (December,1982) recognizes three different access methodologies for local area networks, and actually proposes three distinct standards. 25 Table I summarizes the proposed standards.

TABLE I.

Proposed Standards tor Local Acea rietworks

\begin{tabular}{|c|c|c|c|c|c|}
\hline $\begin{array}{l}\text { hecess } \\
\text { Hethodology }\end{array}$ & \multicolumn{2}{|c|}{ CSMAvco } & \multicolumn{2}{|c|}{ soiken-pus } & Token-RIng \\
\hline $\begin{array}{c}\text { Teansalisalon } \\
\text { Mediug }\end{array}$ & \multicolumn{2}{|c|}{ coaxlal cable } & \multicolumn{2}{|c|}{ Coaxial Cable } & $\begin{array}{c}\text { Notat Pale } \\
\text { of } \\
\text { coarlal cable }\end{array}$ \\
\hline $\begin{array}{l}\text { Eneod ing } \\
\text { Sethens }\end{array}$ & Busband & acoadband & aasaband & Broadband & Baseband \\
\hline $\begin{array}{l}\text { Data } \\
\text { pate }\end{array}$ & $1-20$ raPs & $\begin{array}{l}20 \text { Hags } \\
\text { Pex Channel }\end{array}$ & $1-10$ yazs & $\begin{array}{c}1.5-20 \\
\text { MBPS } \\
\text { per Channel }\end{array}$ & $\begin{array}{l}\text { 1.4 amps } \\
\text { or } \\
-40 \mathrm{raps}\end{array}$ \\
\hline
\end{tabular}

- Tor misted palr Media

tror coaxlal cable Media

Also, in 1982, IBM released the results of a series of internal studies it had made on local area networks. All of the studies focussed on a ring network with token-passing access control and baseband transmission.

So, in summary, the present status is that a set of standards for local area networks is beginning to emerge and the corporate behemoths are clearly divided into different camps. It is expected that the proposed set of standards will be adopted by the IEEE sometime in 1983. It will undoubtedly take several years to determine whether one or another of these standards achieves a dominant position in the marketplace. 


\subsection{Application to Accelerator Control Systems}

Rather than leave it as an exercise for the reader, we
illustrate the application of network technology to
accelerator control system by considering some typical
examples.

\subsubsection{Networking of the Control Center Processors}

The processing components of the control center were outlined and discussed in chapter 3 where they were organized as a number of distinct functional elements: the application program processor(s), the data base manager, the archival and file management system, the manager of console hardware, the program development computer and, perhaps, a large "computing engine" for handling simulations and other compute-bound tasks.

In the pre-networking era of computing, the tendency was to support all these processing functions using one or two relatively large computers. With the availability of modem network configurations, however, other options and possibilities exist. Network technology makes it attractive to configure the central system in a highly modular fashion which distributes the processing requirements over a set of compatible machines, which are interconnected using one of the network topologies described above.

To illustrate the spirit or flavor of this approach, consider the functional decomposition sketched in Figure 4.8, which uses a bus topology and an Ethernet link for inter-processor communications.

One of the pivotal ideas implied by Figure 4.8 is that a particular processing requirement need not, $a$ priori, be confined to a single processor. A given application program should regard the data base manager, the central file system and the computing engine as system resources. Powerful network software structures make it possible to distribute the chore over a set of machines, to manage the synchronization of the individual machines and to provide transparent communication services between machines. 


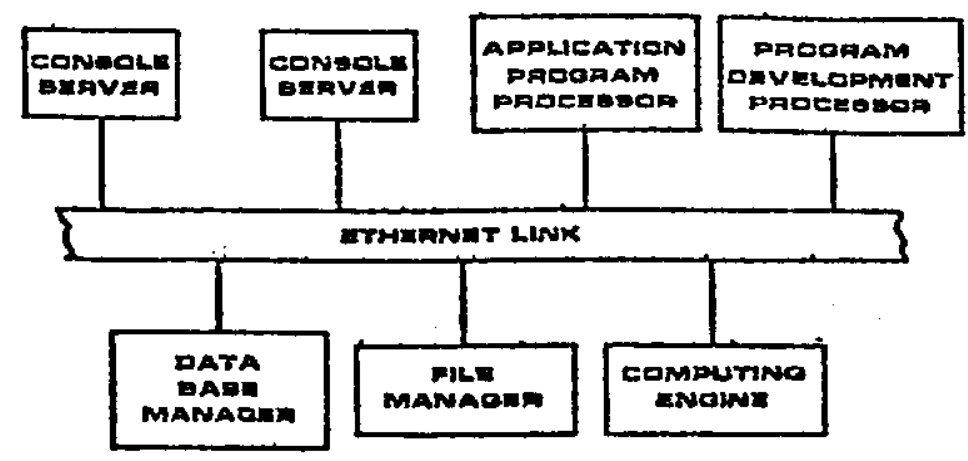

Fiougn 4.

NaTUOAKINO THI CONTADL CENTEA COMBUTEAS WITH AN ETHEANIT LINK

The advantages of such a system structure are immense. It provides the system with a high degree of parallel processing power. It makes it possible to match each processing component of the system to the type of chore it will be called upon to perform. It provides a tool for organizing a system into a set of well definea, modular components.

The single most important advantage of a network-oriented organization, such as that depicted in Figure 4.8 , is a characteristic called its "open-endedness". With a proper implementation it is possible to upgrade the individual modules of Figure 4.8 or to add new processing components to the structure without redesigning the system. An open ended design is one which lasts; individual pieces may become obsolete or inadequate but the design concept, itself, survives the ravages of time. The necessity for and characteristics of an open ended design are so important that they warrant an extended discussion. Chapter 7 treats this topic in great detail.

Figure 4.8 was not meant to imply that a bus topology and an Ethermet link are necessarily the best choice for interconnecting the processors at the control center. Other topologies, other encoding schemes and the merits of parallel vs. serial links all need to be evaluated in conjunction with the requirements and environment of the application. The "best" scheme does 
not require the use of a particular network topology, but it does insist upon a modular structure which offers a high degree of open endedness.

\subsubsection{Interconnecting the Control Center and Local Stations}

In addition to interconnecting the processors at the control center and those at the local station, a more global network is needed to link all the local stations to the control center. This global network will ordinarily have a rather different set of requirements and characteristics than the network used to link the processors at the control center. The global network will often extend over many miles; it will often need to support several hundred nodes, but the required bandwidth for communicating between any two nodes will usually be smaller than it was at the control center.

All of these characteristics argue strongly in favor of a serial transmission scheme using as few cables as possible. (See Chapter 7 on cost considerations.) Given the limited number of cables, it is necessary to manage the available bandwidth sensibly and effectively. A broadband encoding scheme could be quite appealing in this environment. The frequency spectrum could be allocated to provide 5 or 6 high speed data channels, 10 to 20 digitized voice channels, and 10 to 20 video channels for optical surveillance and general purpose communications. In addition to providing the data transfer facilities needed by the control system, one uses the same small number of cables to support whatever voice and video communications might be needed.

There are no fundamental technical conflicts which preclude a system design that lises different encoding schemes or different network technology in different pieces of the system. For example, the system structure illustrated in Figure 4.9 is quite viable.

Here one has a ring network linking all the local control stations and a bus network linking the components of the control center. The two networks communicate with each other using one or more processors which are called a network gateway. The bus network does not need to know about or concern itself with the encoding scheme or access technique used by the ring network, and vice 


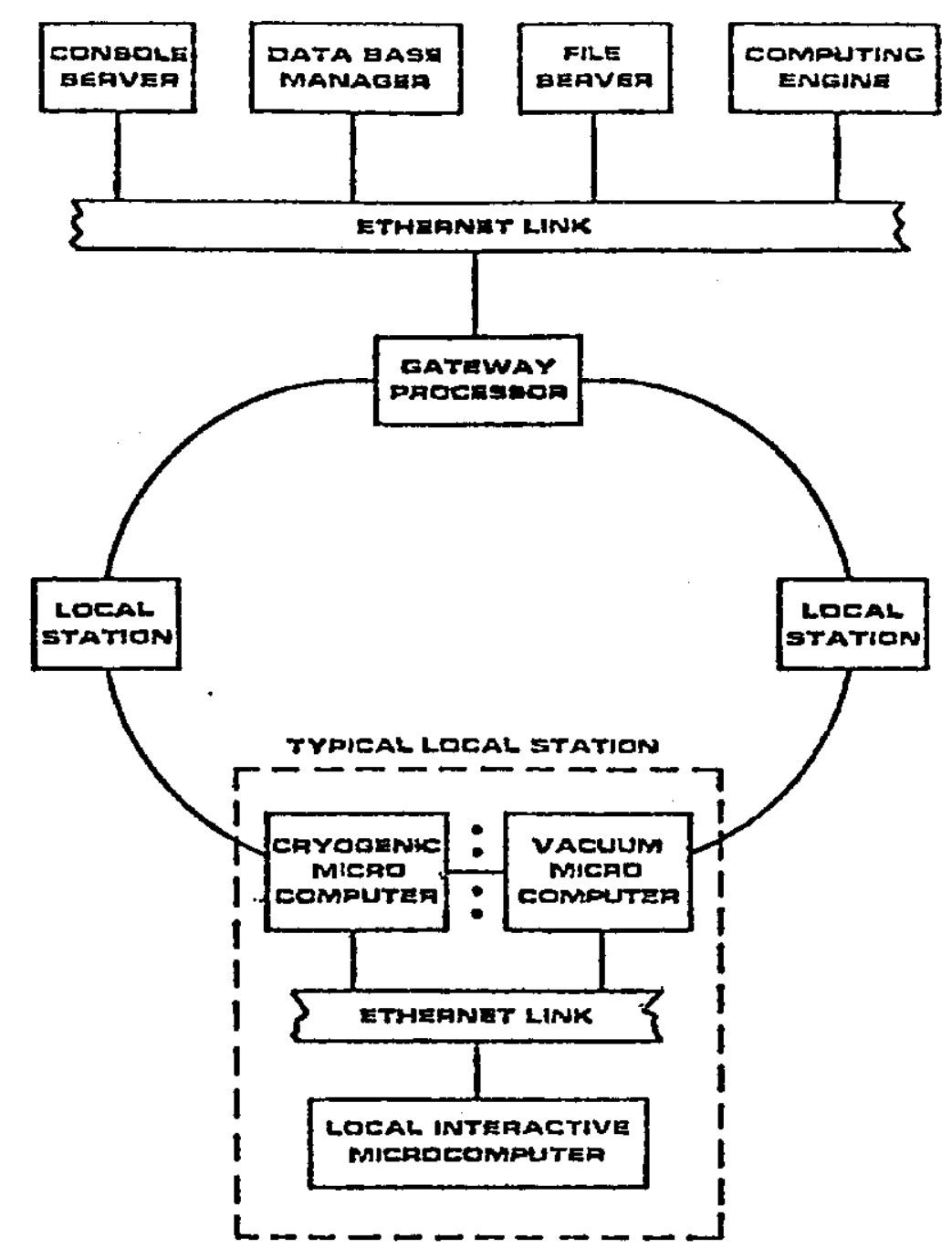

FIGUAE 4.9

NETLYOAK CONFIGUAATION WITH SEVEAAL TOPOLOGIES

versa. The gateway not only serves to connect the bus and the ring but also to provide some degree of isolation between them.

Figure 4.9 illustrates that the application of networking techniques to a large control system can become fairly complex. The burden for applying these techniques in a coherent, consistent and straightforward way is a non-trivial challenge to the control system designer. True, networking techniques complicate the life of the system designer, but they also provide him 
with a tool that allows him to implement a system which has powerful, user-oriented facilities with a design concept that is highly modular and open ended. 


\subsection{Types of Data Base}

5.1.1 The Naming of Variables

Reference to a particular piece of hardware, like a correction element, is by one of a number of methods. To the machine physicist, it should have a name indicative of its function and its position. To the man who installed it in the tunnel, there was another name, which is often painted on the element itself, and which appears in the inventory. In the data base, there may well be yet a third name. From the console, the person operating the machine must be able to refer to the unit by his own name, the details of cross-reference being taken off his hands by the system. In order to do this, the system needs access to a data base.

\subsubsection{The Data Base}

In order to access the hardware with the aim of interrogating or controlling it, it is necessary to get from one or other of its names to a hierarchy of addresses which enable the necessary output instructions to be perfomed. These addresses (for example serial link no., crate number and unit number, as well as a possible address inside the unit) must be associated with the item in the data base describing the equipment.

Numerous other parameters may belong to an equipment: permitted limits on variables, requested values, flags describing which variety of equipment is 
installed, status descriptions and scaling factors are just a few of the parameters which can be held in the data base and updated. The data base is consulted whenever the equipment is changed or examined, and since it is the unique gateway between the equipment and the computer, it can in principle carry a current image of the equipment.

5.1.3 Current Values and the Data-base

The most recently read-back value can be a dangerous piece of information to hold in the data base, for if it is used uncritically it may give a false picture of the current state of the machine. For slow-moving variables this risk is lower, but unless the information "self-destructs" when it is too old to be reliable, or unless it is continuously refreshed by a scan program, there are good reasons for not carrying current read-back values in the data base at 311 . The best ways to discover the state of the machine is to ask the machine.

5.1.4 Program Libraries and Archives

An essential feature of a multicomputer system is a comprehensive system for storing and retrieving programs. This system should be central and unique. A program developed for use at one console should immediately be available everywhere, and a change or correction need then only be performed once. A good version number system can be useful, in case it is required to go back to the previous program. Some operating systems supply this free of charge, allowing the latest version to be used unless otherwise requested. A similar arrangement can be used for creating an archival data base for the accelerator, where sufficient data are stored to enable the machine to be re-started with a consistent set of parameters after a shut-down.

\section{1 .5 The Use of Caches}

When a program makes repeated access to a particular piece of hardware, data base information concerning that piece of hardware may be referred to many times in succession, and the identical parameters may find themselves traveling down the same information highways repeatedly. The attraction in such a case of a lacal cache, retaining the result of the first fetch, is obvious. Circular buffer techniques are available for ensuring that the cache does not overflow, but a more serious problem with caches in the risk always attendant on the existence of a secondary data base. If the 
original data base changes after the first fetch, the cache will be out of date. Something must be done to obviate this risk.

The program using the variable will normally try to find reference to it in the local cache. Failure to find it there will cause the search to be continued in the data base. Clearly if the data base is altered it is important to cause all cache searches to the altered parameter to fail. Various methods of implementing this are possible, and one must be careful not to use a method which will introduce a large overhead of complexity or traffic into the system. One of the simplest arrangements is simply to clear all caches after any change in the data base. This may be sufficient when the data base is changed rarely.

\subsection{Time-Critical Operations}

\section{2 .1 Interrupt Methods}

Some events are out of the control of the computer system. A person hitting a key, a device changing status, or the end of a slow requested operation like an $A-D$ conversion will require subsequent attention from the computer. One way to service such a change of status in the outside world is to perform regular scans which poll for them. Another is to use the interrupt system of the computer. Polling will either be done at regular intervals or when the computer has nothing else to do. If the latter, then it is exactly equivalent to the use of the lowest priority interrupt. If a higher quality of response is required, it is important to note that this corresponds to a need for privilege over other processes served by the same computer, and that such privilege cannot be accorded to more than one user at any given instant. The dictates of good design indicate arrangements in which computers need not be interrupted in a hurry. Such arrangements are possible, provided that for each task which is time-critical a processor is supplied which is dedicated to that task. This processor can do other work, but not other priority work.

\subsubsection{Synchronous Processes}

A typically time-critical process is one in which a large number of readings must be made at widely distributed places simultaneously. No method dictated with precision from a central location will do this work, as the requirement at each measuring station is essentially a high priority one. The solution is to 
provide sufficient hardware for parallel processing to be performed.

Each measuring station must be equipped with buffer memory, a centrally synchronized clock, and local intelligence capable of receiving instruction from the computer center, sequencing the local operation, and returning the result to the center. The measuring stations are then told, at leisure, to prepare to make a measurement at a given instant of time. The instant, which is the same for all stations, is well into the future so as to ensure that without any hurry all stations shall have received their instructions in time. The central computer can then go round, at leisure, collecting measurements which are known to have been gathered simultaneously.

Most time-critical processes can be implemented by variations of this method. A typical problem is a family of function generators driving programmed power supplies around a circular machine. If all generators have double buffers, one to hold the function currently being generated and one to hold the function to be used next, switchover from one to the other may be made simultaneously at all locations.

Simultaneity is achieved by piping some sort of clock around the site. Since the exact timing of the event is often associated with a specific point in the accelerator cycle, it is often useful for the clock to specify events in accelerator time rather than in clock time. The clock line may also carry event identifying information. Events can then be re-located in time or even inhibited, causing identical effects at all locations which depend on them. This gives considerable control and flexibility.

\section{$5 \cdot 2 \cdot 3$ Limitations}

The real-time methods described above take time-critical operations off the critical path. It is important to note that the work of transporting the information may still need to be done on a restricted, if less critical, time scale. Thus in a cycle-by-cycle process, double-buffering and independently timed acquisition may trap the information at a precise point of the cycle without real-time aid from the computer, but unless the reading is taken out of the buffer by the same time next cycle, the buffer is likely to be overwitten 


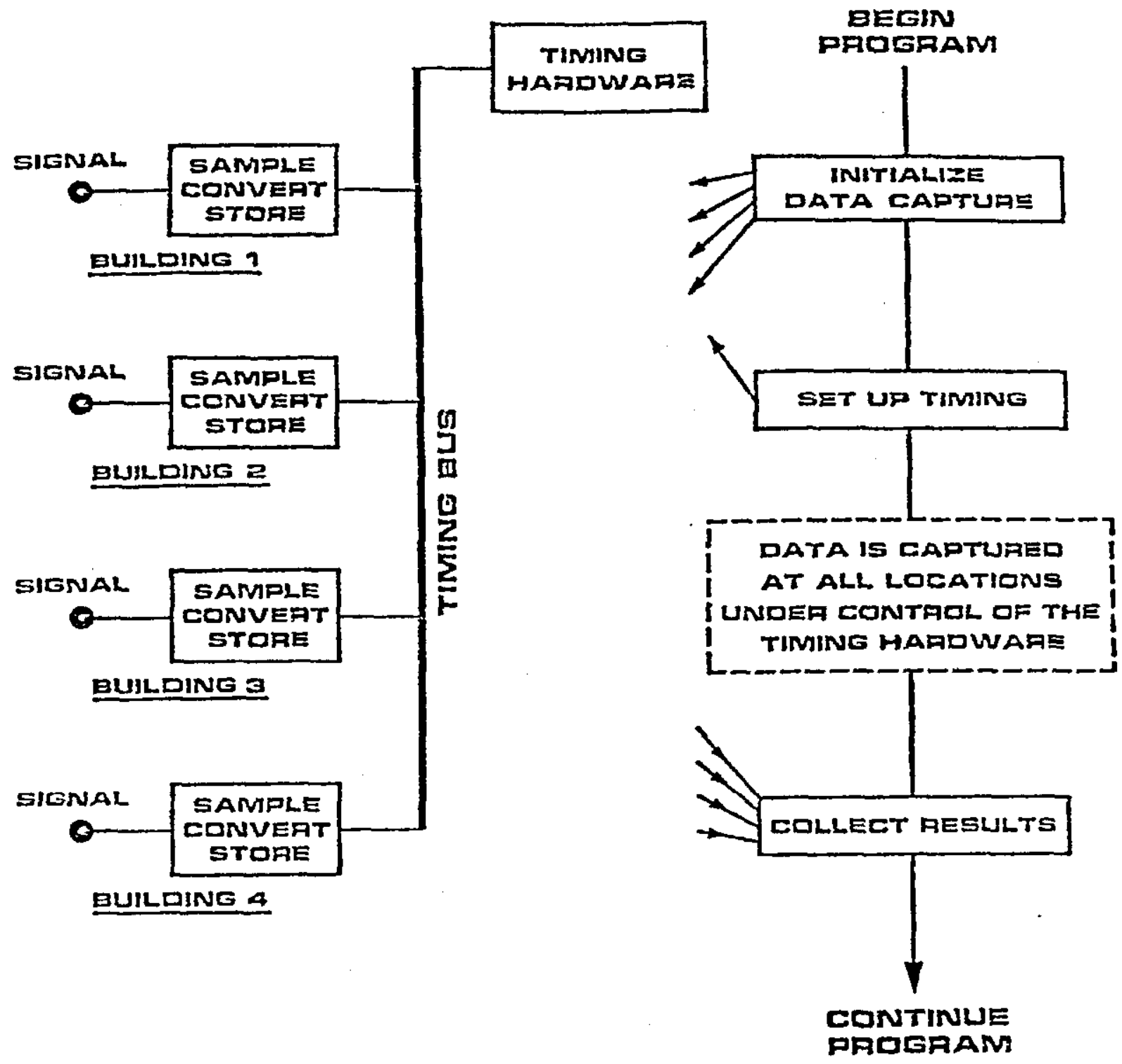

FIGUAE 5.1 SYNCHAONOUS DATA COLLECTION.

by a new value, and the computer is therefore linder pressure to fetch all readings before the next cycle is too far advanced. The time-criticality has therefore been relaxed from milliseconds to seconds, but it is still there.

Peaks in the computer activity, which make it difficult on occasion to get all the readings processed 
in this way can be ironed out by supplying additional hardware at each station, that is multiple buffering the readings instead of double buffering. None of this, of course, will compensate for a computer system which is inherently too slow to process the total data as fast as it is being generated over the long term. You may need a more powerful computer to do the work!

5.2.4 Is it all critical?

If valuable resources like high priority levels in a computer are used, it is important not to misuse them, as this may degrade the rest of the system to no advantage. A high priority program should perform its high priority task and then immediately allow tasks of lower priority to take the computer. Usually only the acquisition of the data is time-critical, and further processing can be done at leisure, while the computer is free to process other people's high priority interrupts. Similarly, if special hardware has been installed for a time critical operation, it should only be used for less time critical work in addition if it would otherwise be truly idle.

\subsubsection{Post-mortem snapshots}

A prime user of valuable bandwidth is data logging. Much data-logging consists of the transfer of information to a central location, where it is recorded in case it is needed. In many cases it is preferable to record the information locally, only fetching small amounts to the center selectively when necessary. This need often occurs only when the beam crashes or a superconducting element goes normal, and in this case the information does not even need much local storage. A circular buffer, used repeatedly, serves to hold the most recent sequence of measurements, and in the event of a crash or quench this buffer, and its fellows in other locations, serves as a "snapshot" which can be examined selectively to investigate the state of the machine in the last morents before the event in question.

\subsection{Protection Requirements}

\subsubsection{Hard-wired protection}

Anyone can make a mistake, and so can machines. Electrical protection is as old as the commercial use of electricity. Fuses are used to protect circuitry, and door interlocks to protect people. Even when the undesired condition is known to the computer and it would in principle be possible to prevent undesired 
consequences by computer control, this is usually unwise. This is often confirmed by the legal codes, which require interlocks protecting personnel to be mechanical. In general, protection devices should be mechanical and as simple as possible, to minimize the risk of failure.

This is not to say that the computer has no part in this kind of protection. If the mechanical devices are well supplied with computer connections, and the status of all relevant machine elements is available to the computer, it is not only possible to ensure that the operator knows which interlock has tripped and why, but it may even be possible to inform him that a certain operation will result in an infringement of the safety conditions and an interlock trip. This kind of thing is particularly useful, for example, when the conditions for personnel access to a beam line area are dependent on a large number of variables (certain high voltages isolated, beam stoppers in place, radiation levels checked etc.). In a case like this, the operator can consult a screen to check whether access is feasible or whether conditions still exist which make it impossible. If in spite of this he attempts to allow access to an unsafe area, there is always the hard-wired protection system as an ultimate resort. Furthermore, the operator who has made this potentially dangerous move is not only protected from its consequences but informed by the computer that the interlock hardware has operated.

\subsubsection{Remotely Controlled Devices}

Devices in the field which are remotely controlled from a control center often have local controls as well. In addition there may be a local-remote switch which gives a choice of location from which the device can be controlled. Problems of personal safety are involved, as well as operational ones.

Some devices can give rise to an actual hazard when switched on. Electric motors, valves controlling fluids and power supplies attaining lethal voltages all need to be isolated so completely that remote control cannot override the isolation, if personnel access is to be permitted. The ability to isolate the equipment without this safeguard can be a dangerous one.

Less dangerous, but nevertheless bad for the operational integrity of the system, is the ability to control equipment from more than one place simultaneously. To address equipment from one place when it is currently being used from another can be a 
nuisance, frustrating, and may even cause both people's instructions to be disobeyed. The latter problem is usually taken care of by the operating system, simultaneous requests being served correctly in sequence. If one person switches the device on from one console and another switches the same device off from another, this might be considered annoying or unacceptable. Different accelerator installations have different opinions on this. Some have elaborate arrangements where a particular console pre-empts a whole group of control variables and all other consoles are forbidden to use them. Some have personal access codes for responsible people and trust such responsible people not to interfere with each other.

It all really depends on the number of consoles, their geographical locations, and the caliber of the people who will have access. Protection schemes can be a nuisance to people doing something unusual if they are too safe!

\subsubsection{Protection of files and data bases}

Information should be protected, whether it comprises programs, data or even elements in a database. Various methods are available for this, depending on whether protection is needed by teams against the outside world or by individuals.

If the files are stored under the operating system of the computer, there will usually be a password scheme which can be used directly. It is not difficult to devise a scheme which protects hardware write operations with the same password, but unfortunately this is not good enough. Whereas it is usually satisfactory for a file to be attributed to an individual and to forbid everyone else to use it, the same is not usually possible for a control variable. Whoever is currently operating the machine must be able to change all its variables, and if there is to be a protection scheme, access must be by programs or by privileged consoles. The CERN SPS has an elaborate scheme in which access is defined by team, and the rights of the team member currently operating are in effect specified with every equipment access and checked to see whether that access is permitted. The man who builds power supplies can specify the permitted upper limit on the voltage of the power supply. No one else may change this limit, but every qualified operator may switch those power supplies on and off. At the CERN PS, no variable may be changed without first reserving it, and while it is reserved no one else may change it. This 
is simpler. At Fermilab protection is by control location only, and all central control consoles have universal access, whilst remote stations can be prevented from making alterations by program control. This is even simpler and it seems to suffice.

The lesson to be learnt from all this is that protection schemes can be made arbitrarily safe and complex, but that intelligent operators can work quite a simple scheme, or even with no protection at all.

\subsubsection{Breakdown of the control system}

All major accelerator installations aim for the characteristic that a breakdown of the computer control system does not affect the machine. A loss of control need not necessarily lead to a loss of function, and it is quite practicable to design the accelerator in such a way that it will go on pulsing and extracting, or storing, in the predetermined manner, even if communication with the control center is lost.

To achieve such independent operation, all values for digital-analog conversion must be held in registers outside the control system, and all cyclic functions synchronized by the timing system must be generated by autonomous hardware. Care must also be taken that loss of power in control system elements does not send spurious impulses to these accelerator elements and spoil an otherwise perfectly reliable system at the moment when control is lost.

Closed feedback loops involving the computer system, such as those used in the control of spill structure in an extraction system, are permitted, so long as they are designed with failure to safety in mind. Even when the accelerator has been working for some years, the system should be studied from this point of view from time to time, to ensure that these criteria have not been violated during changes and improvements.

If these precautions are taken, it is possible to have an accelerator which, on the breakdown of the control system, slowly degenerates due to drift in the critical steering elements. With luck, control may be re-established before this drift has made it necessary for the hardware interlock equipment to shut down the machine. Typically, a period between five minutes and half an hour can be made available by these methods to repair the control system on a running machine. 


\subsection{Pulse-to-pulse Modulation}

A problem which was first seen at the CERN PS but will in future occur at other accelerators, is that an accelerator can sometimes be used for two or more purposes, not sequentially by retuning, but in a fixed repetitive supercycle. Thus a pattern of successive CERN PS pulses, called a "PS supercycle" was used to do fixed-target physics, fill the intersecting storage rings, and act as injector for the CERN SPS. Similarly the Fermilab Linac is used for cycles of fixed target physics with the Tevatron, for cooling-ring studies and for producing low energy neutrons for radiation therapy, in a regular supercycle of alternating pulses.

The requirement here is twofold: the timing system must clearly supply information to determine which part of the supercycle is currently being executed. The function values and setting parameters which give the machine its characteristics during each cycle must be remembered in memory areas which are particular to each cycle. This replicated memory may reside either in the computer, or in the actual microcomputer doing the function generation. If the system was originally built without pulse-to-pulse modulation in mind, the microprocessor arrangement is usually the simplest one to add. In either case the timing system will dictate which of the memory areas to use to generate the functions and settings for a particular phase of the supercycle.

There remains the problem of tuning a machine being used alternately for different purposes. We might consider that we have two or more distinct "accelerators", each of which must be tuned individually only during its own phase. Clearly what is needed here is the ability to access only one phase at a time from the console, so that the operator is for the time being oblivious of the existence of the other "accelerators". This can be done by the simple stratagem of waiting for the right time whenever a read or write is done, so that only the appropriate parameter is changed or displayed. Suppressing the currently irrelevant phases from view or access requires a much more sophisticated re-entrant programming scheme: it is done at the CERN PS, where a completely general arrangement allowing a supercycle containing up to 64 cycles of 8 kinds is available, with complete protection of any "accelerator" from all others. 
CHAPTER 6

\section{SOFTWARE}

\subsection{Application Pragramming}

6.1 What are application programs?

Some programming is concerned with the transfer of messages, the protocol of hardware drivers, and the implementation of the operator interface. This kind of programming can be called system programming just as surely as the compilers, loaders and operating systems.

Application programing is that work which is concerned more with the accelerator than with the control system. The programs serving applications tend to be of two kinds: those that deal intimately with individual equipments or subsystems, and those which provide a human interface. A large part of the programming work involved in a large modern accelerator is concerned with applications programs.

The programs which are near to the equipment being controlled tend to be used either for local processing and checking, or to provide a convenient interface between the accelerator hardware and the programs in the control room. If there is any local computer control this is included here. This kind of application program has a tendency to be written in low-level code, to require an intimate knowledge of the hardware, and to be very time-consuming.

The programs which provide the human interface are also those which perform any necessary calculations. 
These are what people think of when they talk about control programs.

\subsubsection{Programming: Public or Private?}

The application programs, as described above, can require a large gamut of skills. It is difficult to find people who are expert at everything from theoretical machine physics, through the machine code programming of real-time processes, right down to the signals on interface highways. It is not even practical to design a system which relies on the help of a number of such polymaths. In practice, it is more usual to let people pursue their own specialties, and to split the work up and allow people to work within their own fields.

Here there are two schools of thought: Application programming can be done by programming experts, effectively working under contract to an applications expert. Some very good collaborations have existed in the past, where for example a machine physicist works well with a skilled programmer to produce programs to measure for a commissioning program, or a programmer helps an electronic engineer to commission a piece of interface equipment.

A variant of this one-to-one arrangement is one in which the application expert writes a very precise specification of his problem and gives it to a specialist service for programming.

The alternative to this is for the computer experts to work only on methods of making their system easy to program and use, and for the application experts to do their own programming in a simplified environment prepared by the computer people. The modern tendency is to do it this way, with varying degrees of success at different installations. In control centers, it tends to be practicable to design easy-to-use, well documented facilities for data acquisition and the operator interface, such that an engineer-in-charge or machine physicist can program his own application program. Such programs tend to be very useable, and with sufficient support from the computer people they can be quite efficient. The work of writing them is stimulating and creative, and many accelerator experts can be easily persuaded to contribute to the effort.

Far less success has so far been demonstrated at the other end of the control system. Here the work is much more technical, being aften concerned with real-time 
acquisition and high-speed scanning. Much of the work is done in machine code on microcomputers, and the dictates of efficiency preclude more user-friendly arrangements. This end of the system is therefore still in the hands of the computer expert, and is therefore not as useful to the physicists and engineers as it would be if they could be given direct control of it. Since the problem is mainly one of speed and efficiency, the advent of cheap fast microcomputers may allow "democratization" at this end too.

\subsection{Languages}

\section{2 .1 Choice of language}

The choice of languages is influenced by a number of factors, the least of which is suitability or efficiency in the context of the program which need to be written. If, for example, a large number of the people available as helpers are already FORTRAN users (as is the case if they are scientists helping out with the control system) one might do well to use FORTRAN in spite of the defects listed below. If the computers being used have very good PASCAL software, one might be tempted to use that.

Languages are in general of two kinds. Those where the original program text is interpreted and obeyed line by line, (interpreted languages) and those where the original text is translated into machine code by the computer, the machine code being obeyed subsequently in a second phase (compiled languages). The tendency is for compiled languages to be harder to use but for the code they generate to be very efficient, compact and fast, and for interpreted languages to be easy to use but very slow. Factors of ten in speed are not unusual. The choice of language is complicated by the fact that between pure compilation and pure interpretation there is a whole spectrum of operational possibilities, with language implementations existing at all stages.

What are the high-level languages available on most computers?

\section{$6.2 \cdot 1.1$ Fortran}

This is the commonest. Ideal for large mathematical codes, which do not represent a major part of the programing load on a control system. Fortran can only be compiled, never interpreted. A very comprehensive subroutine-calling arrangement allows large programs to be well-structured. The 
code itself is neither very easy to read nor very safe by moderm standards. String-handling and list processing are not very well provided for by modern standards, and both these are features needed in coding a control system. The ability to call subroutines written in assembly code is a redeeming feature of FORTRAN.

\section{2 .1 .2 Pascal}

Pascal is a language which was ariginally designed as a teaching language, but has since been adapted and extended for most computing needs, being an effective alternative to FORTRAN in all its applications. It is available on most computers and micracomputers which have FORTRAN. Much of the structure of Pascal involves an attempt to make the user specify what he intends, in circumstances where the FORTRAN compiler would make assumptions for him. This means that a Pascal program needs a lot of explicit definitions, and these must be repeated, or at least invoked, in each procedure or subroutine. Thus Pascal source programs tend to be bulky and verbose, which frustrates people used to FORTRAN. Since all this specification is required to enable the compiler to check for inconsistencies and determine what the writer intends, Pascal can give rise to very efficient reliable object code.

$6.2 .1 .3 \mathrm{ADA}$

This is the Department of Defense standard language for application programs written as part of government contracts. It is based on Pascal but is more comprehensive. Subset compilers are not permitted, but there is nothing to prevent a user voluntarily restricting himself to the use of a subset of the language, which is a good thing, as there would otherwise be a long obligatory learning procedure. Compilers for ADA will in due course exist for all large computers commercially available in the US, and these will produce object cade for all computers of any size. It is difficult at present to find people with experience of this language, but anyone planning a large control system must obviously take ADA seriously.

\section{2 .1 .4 BASIC}

Originally developed as a teaching language at Dartmouth College, BASIC has become the unique 
universally accepted language for interpretation. The original basic is very limited, with short variable names which are not mnemonic and few features suitable for anything but arithmetical work. It is a difficult language in which to write long and elaborate programs, as subroutines are numbered, not named, all variables are global, so that it is impossible to borrow a working subprogram from someone else. However, extraordinarily developed interpreters of BASIC type exist on various computers. Many of these have features for graphics, calling of subroutines written in assembler code, and even provide the string and list-processing facilities essential in control work and absent in many compiler languages. BASIC is easy to learn and easy to use, and is very suitable for certain kinds of control work. Interpreters are even easier to write or improve than compilers, which is a strong temptation to use them. They are, however, very slow, and the system designer who uses an interpretive language must realize that he is restricting himself to a different kind of control system; one based on small, simple application programs and not relying on extensive program libraries, and one which is either content with lower processing speeds and smaller information flows or which uses elaborate parallel-processing methods to offset the inherent slowness of interpretation. There exist compiled BASICs and intermediate forms called incremental compilers, which attempt to offset the inherent slowness. The great advantage of BASIC and its associated languages is ease of learning and use, as well as the fact that the functions of loaders, editors and other system utilities are all part of the one process. Systems based on the intelligent use of interpreters allow many people to be recruited for the work of writing application programs, and this may be an overriding argument in their favor.

$6.2 \cdot 1.5$ NODAL

The NODAL26 language is worth individual mention here, as it is an essential component of the control system at the CERN SPS and that projected for LEP, and is also used, in various variations, at the CERN PS and at various smaller European installations.

NODAL is an interpretive language like BASIC, but the statements, instead of being labeled with integers, bear labels with two numbers after the 
decimal point. Groups of labels having the same integer value constitute paragraphs, and the paragraphs can be referred to by this integer. Thus in a program with the labels:

$$
\begin{aligned}
& 1.00 \\
& 1.15 \\
& 1.20 \\
& 2.30 \\
& 2.40 \\
& 2.80 \\
& 3.15 \\
& 3.25
\end{aligned}
$$

The statement DO 2, placed anywhere in the program, will cause the middle paragraph (statements $2.30,2.40,2.80)$ to be performed, followed by execution of the statement following the DO 2. This concept of groups came from a language called FOCAL, first introduced by Digital Equipment Corporation.

This group structure enables NODAL to be used for the construction of highly structured programs. There are mechanisms for allowing the selected paragraph to be executed in other computers of an attached network, and also for allowing construction of arbitrary NODAL statements during execution. Since the language also contains string-handling features and real-time hooks (WAIT-TIME, WAIT-CYCLE), elaborate data-collection processes involving several computers are easy to devise in a few statements.

NODAL also contains hooks for elementary graphics and has a very strong diagnostic capability with on-line editing at error time. All this, of course, costs time, and most NODAL implementations allow the embedding of compiled FORTRAN subroutines at speed-critical points. The SPS-LEP complex is the only one extant which relies almost exclusively on an interpreter, and there is some discussion of whether this is due to some inherent property of those accelerators or whether it is not applicable to other structures. Most other installations that use NODAL or its derivatives rely on it as an aid, rather than as the major tool for the construction of control programs. It is often claimed that certain accelerator parameters or characteristics, such as the repetition rate of a fast-cycling machine, preclude the use of an interpreter as the primary 
language for writing control programs. However, most time-critical operations can be handled without placing excessive requirements on the choice of language (cf. Sect. 5.2). In view of the fact that this approach allows good programming at the most sophisticated level by relatively unskilled people, and that it has been so successful at CERN, it is surprising that this method has nat been relied upon in any large subsequent projects.

6.2.1.6 Assemblers

Assemblers are efficient. All computers have them, and in principle all application programs can be written in assembly cade. With strong discipline, the resulting programs can be well structured and well documented. Undoubtedly a small team comprising one or two high-powered software experts can program a compact control system completely in assembly code.27 Numerous examples of successful systems of this kind exist, usually involving one or two computers. Even at this level, the resulting system is unduly reliant on the continued attention of the builders, but in a small, closed environment this can sometimes be tolerated. It is important for snyone contemplating the design of a system programmed in assembly code to be aware of these limitations and be prepared to live with them. The system cannot easily be extended, cannot easily use newly recruited staff, and must be well designed and implemented by people who are committed to maintaining it themselves until it is obsolete. With these restrictions, assembly code can be used to program a very efficient, small control system. It should not be rejected out of hand because it is not "modern".

$6.2 .1 .7 \mathrm{C}$

The $C$ programming language is a relatively "low-level" language developed at Bell Laboratories in the $1970^{\prime} \mathrm{s} .28$ It is particularly attractive as a system development language, although it has been used for applications involving numerical processing, text processing and data base programs. The language itself (and its associated compiler) provides no input-output facilities, but rather relies upon a "standard" input-output library which provides an interface to the operating system.

The $C$ language contains a very rich set of operators and very powerful and flexible facilities 
for manipulating pointers and performing address arithmetic. Because of these features, $C$ is best described as an intermediate-level language lying somewhere between an assembler and a high-level language like FORTRAN or PASCAL. In many situations it can provide an attractive alternative to the use of assembly language. Since the $C$ programming language is machine independent and relatively easy to learn, its use does not demand the level of expertise required with an assembler, and its programs tend to be highly mobile and portable. On the other hand, the $C$ language is not yet well known by the physics and engineering communities. It will be interesting to see whether or not its probably unique role as an intermediate-level language promotes its popularity in the coming decade.

\subsection{Aids to understanding}

\subsubsection{Dn line documentation}

A large modern control system will probably be faced at times with the arrival of staff who need to learn either to program or to use the systern. Good systems contain computer-assisted aids to such learning, and it is sensible when planning a control system to apply some fraction of the effort available to incorporate such aids. In addition, if the system involves a lot of computers and a lot of people, it may be better to keep the documentation in the computer than on paper, since the computer documentation can be kept up to date more easily and referred to from numerous locations, whereas documentation on paper can be out of date even when more up to date versions exist.

If a large commercial operating syster is used in the computers, it may well contain "mail" and "help" facilities which can be used for some of this work. otherwise the system designer must purchase sufficient bulk storage for documentation and design something himself.

A global "help" facility can enable the user to request the display of a screenful of information at any point. Such a facility can be incorporated as an item on every page of the menu or touch-button tree, or even as a dedicated button. If it is so designed that it is easy to add to the text during the operation phase, the responsibility for producing this text can be left to the first generation of operators, and subsequent generations can then add small notes clearing up points which were 
badly explained in the first edition. Such an arrangement is preferable to one in which the original programmer writes the "help" page, since the programmer is very unlikely to be aware of what an operator will find hard to understand.

Some programming aids, too, can be supplied at the console. Very clever aids to the design of graphics displays and touch-button layouts can be designed, using the ball and cursor and the keyboard. When a program has been written and tested in free standing form, it should be possible to add it to the touch-button tree or the page structure by means of an interactive operation. Similarly a page displaying and controlling variables should be built up by interaction, and the addition of new hardware should not require programming, but should be possible on-line at the console. All this is easy to implement if the original programs are table driven, so that changes can be wrought simply by altering the tables.

\subsection{Programming the microprocessors}

6.4.1 Are microprocessors different?

The programming of microprocessors presents a slightly different problem from the programing of the larger computers of the control complex. This is not so much a question of computing power (the latest microprocessors are just as powerful as many minicomputers) as of physical structure and the availability of peripherals.

The actual programming can be done either in assembly code or in one of a number of higher level languages mentioned above. If the translation from this to an image of an executable program in the memary of the microcomputer cannot be done in the microcomputer itself, and this is usually the case, then it must be done elsewhere. The resultant rachine code is then down-loaded to the memory of the microcomputer. There are two methods of doing this: one can use a development system containing the necessary assemblers, compilers, loaders and other programs needed to translate and load the program; alternatively one designs the microcomputer in such a way that it may be down laaded through the control system, and then uses suitable software in a minicomputer of the control system to translate and down load. There is a growing tendency to use the latter arrangement. Computers tend in any case to be on busses fed from the control system, and more and mare of the 
necessary software exists on minicomputers for all the important micros on the market. The ability to recompile and down load a new version of the microprocessor program, and then proceed to test this new version without leaving the accelerator console, can be very valuable.

\subsubsection{Freezing the program}

Once the program has been written and tested, it is theoretically possible to replace the random access memory (RAM) containing the program image by read-only memory (ROM). The advantage of this is that the program is then incorruptible: it does not need to be reloaded after a power failure, neither can it be "improved" by a well-intentioned worker. In principle, then, a program in ROM makes the equipment containing it into a special purpase device, and fixing the program in ROM is analogous to hard-wiring a logic circuit. Changes must then be performed by mechanically replacing each ROM unit. On a large machine this can require a visit to each location in order to install the new memory unit, and can be rather tedious by comparison with sitting at a console and updating all the RAM memories by computer down loading.

The ideal situation, of course, is to have a program frozen in ROM, so perfectly designed that all possible variants in the requirements can be satisfied by making changes to an array of parameters kept in RAM. New modes of operation can then be effected by down loading parameters, while the program itself never needs to be changed. A suitable interpreter in ROM, with the interpretive statements in RAM, is of course a special case of this.

The biggest advantage of keeping programs in ROM is that once the program has been debugged and installed in this form, one is no longer reliant on the presence of the expert who wrote it. The computer is installed as part of a piece of fixed hardware with a guaranteed regimen of operation, and if anything goes wrong we replace the microprocessor or its memory. Never individual words of memory. Many instruments and household devices are nowadays abtainable which use microcomputers in this way. You never call the man who wrote Pacman when your game machine goes wrong! 
CHAPTER 7

DESIGN ASPECTS

\subsection{Introduction}

In this chapter we discuss some of the things which have to be thought about when designing and planning a system. These are management considerations which considerably influence the general architecture of the system as it is conceived. We shall discuss the effect of available money, of time-scales, of personnel needed to execute the project, and of the technical and industrial environment. In judging which of a number of available techniques to use, or how to lay out a system architecturally, a number of compromises must often be made on the basis of the above criteria. It is therefore important to note that there exists neither a correct control system nor an optimal strategy for building one. The best that can be achieved is a system which works; which is built within its budget manpower and timescale estimates, and which is reasonably amenable to subsequent changes in the requirements and the environment. We shall discuss below some of the factors which must be correctly assessed to achieve these aims.

\subsection{Costs: where does the money go?}

\subsubsection{Computers and interface chassis}

At one time, computers represented the most important part of the cost of a control system. An "economy of scale" argument was used to justify the purchase of a very few powerful machines which could time-share the load. In recent years the picture has changed, and a given number of instructions per second is 
more expensive when performed by a single large machine than by many small ones. Unfortunately, good operating systems tend to be supplied only with the larger machines, so a compromise must be made. Ideally one might try to specify two biggish minicomputers with full operating system and peripheral facilities, and a large number of compatible microcomputers or small minicomputers with simple but adequate software. The bigger computers can then be used to support compilation, data bases and printers, while the small ones are used for control.

Crate-and-bus systems are discussed in detail in Chapter 2, but it should be mentioned here that they can represent a very substantial proportion of the control budget. The proposed LEP control system actually builds the computer cards directly into the crate-and-bus system used for interfacing, in an effort to reduce the number of physical units in the system. 29 This may be the wave of the future.

\section{2 .2 Cables}

Since by definition we are discussing large control systems, an important factor in the costing is the price of cable. The price of a cable depends, of course on its quality and on the length installed, but is important to note that these are not the only factors in the cost. The cable must be laid and terminated at both ends by plugs or connections of some sort. Even for quite long cables, these two operations can seriously influence the cost per unit length. There is an important nuisance factor when it is required to add a single cable to a system of cable trays already equipped, and the incremental cost of an extra cable may equal that of several cables at the time of installation.

Termination of a cable is a skilled, error-prone process, if the cable contains many wires. Noise suppression and the rejection of common mode potentials can often require specialized circuitry at each end of the cable, and again the replication of this circuitry for each of a large number of lines can outweigh the cost of the cable per unit length.

There are therefore many arguments for spanning large distances with very few cables, each having considerable bandwidth and carrying many channels. The very elaborate equipment needed at each end of such a multichannel trunk must be considered an investment whose cost is shared over all the individual communication 
lines provided, and the cost per unit length of the entire complex, comprising cable, terminations and multiplex-demultiplex circuitry, must be evaluated on a global basis. The argument for serialized transmission will usually be self-evident. In addition, serialization makes it economically practicable to consider the use of fiber-optic techniques, which give a high bandwidth per link and can save money on the noise rejection and common mode protection circuitry at the ends.

It is important, of course, not to take this trend to few conductors so far as to put all one's eggs in one basket. While it is obviously easier to check and repair few terminations than many, a fault in a wide-band system will be catastrophic, not partial. A system which relies entirely on multiplexed communication must contain duplicated resources, preferably with an automatic mechanism which causes replacement or graceful degradation on the failure of a transmission.

The entire foregoing argument is null, of course, when one end of the cable is obligatorily in a radiation zone. Since the terminating electronics cannot survive exposure to radiation, individual cables must be brought out to a place where multiplexing is possible, and the cost of these cables and of the space for accommodating them can be so considerable that it may even influence the choice of number and position of the enclosures containing the electronics.

\subsubsection{Distribution Panels}

The heavy-duty cables which are used for long runs are often terminated on panels or connection boxes before being joined to lighter, electronics-type wiring for connection with the equipment. Provided such a panel is passive, it only adds the cost of performing the extra joins, and even this is paid for by a certain gain in flexibility.

As soon as this connection is active, however, it has to provide de-multiplexing, modem facilities and optical isolation, and therefore requires electronic circuitry and power supplies. To first order, all boxes containing power supplies and electronics cost about the same amount of money, and therefore the insertion of an additional box containing active circuitry should preferably be avoided. If at all possible, therefore, the cable spanning large distances should terminate in the crate which will treat the information, so that the fractional overhead cost of each cable connection shall 
be minimal. The need to be able to terminate and deal with outdoor cables and the signals from them may influence the choice of the crate system used for local information treatment.

\subsubsection{Software costs}

It used to be thought that software was free. In recent years it has become generally recognized that software not only costs money, but that bad or unmaintainable software can be responsible for cost overruns, missed deadlines and even total project failure. The strategy for writing the software must be planned together with the hardware architecture, and the arrangement decided upon must be such that an accurate and preferably low prediction of the software costs can be made.

The use of "well-structured" software is now known to be beneficial. Individual modules with conceptually simple definitions are linked through well defined interfaces to form a larger program which can be understood without considering the detailed design of the modules themselves. What is less well know is that in a very large system considerable economies in effort and complexity can be attained by a careful choice of what individual modules to build.

Software modules which can be purchased or otherwise obtained are usually worth incorporating. Such modules will in all probability do a lot more than is required, and will include the functions needed only as a subset. This is particularly true of operating systems, where however assembly options may be available to remove some of the unnecessary features. In any case, purchased software will often be more expensive in resources and speed than custom-made software, but this is no reason to reject it. The judicious choice of an architecture allowing parallel computing will usually overcome both defects of the purchased product, and the arguments in favor of home-built software are always seen to have been weak when the project is viewed with hindsight.

Even if the principle outlined above is followed, and a system is built using as much existing software as possible, there will still be a lot of custom software to write. Here a valuable architectural stratagem is available which is generally accepted in engineering but less so in software planning: mass production. 
Mass-produced software has the same advantages as mass-produced hardware: low overall design costs, production-line efficiency and quality control. In software, it consists of the conception of general-purpose designs which can be customized for various applications, by being table-driven or having a suitable customizing subroutine attached. Ideally then, it should be possible to design a control complex in which each computer contains an assemblage of commercially available modules, modules drawn from a carefully minimized pool of locally designed ones, and a minimal amount of customizing information in the form of parameters and subroutines. The choice of the modules to be used as part of the project is a critical one. A good general-purpose hardware driver, perhaps. A handler for device-dependent data bases or for the transmission of inter-computer instructions and data. Graphics. An alarms and limits program which will be available in many processors, and so on. It should be noted that the most flexible of all table-driven programs is an interpreter, where the instructions can be considered parameters and the interpreter a parameter driven program.

A system comprising units of this kind is particularly easy to maintain and improve. Often a change of parameters is sufficient to obtain a new function. In more extreme cases, an additional computer with a combination of existing software modules can allow the installation of a new subsystem with a minimum of software effort.

\subsection{Planning and the Setting of Time-scales}

A number of factors have to be taken into account when deciding on a schedule. The building of an accelerator takes several years, and while it is necessary to take advantage of this time to do design work on the control system, there is no point in building control equipment so far ahead of time that it is obsolete by the time it is needed. The physical installations, such as cable trenches and buildings, may not yet exist. On the other hand, small pieces of the control system are needed for commissioning the individual components.

It is therefore important to design a system in which local commissioning of subsystems is done with individual computers, and the whole can be integrated into a large control system subsequently. If the possibility exists of designing the system in such a way that local commissioning programs can subsequently be run with minimal change from the control room, so much the better. 
It will therefore be seen that in the planning stage, the local equipment interfaces, the computers to be used and the application program languages must be decided quite early on, whereas the network aspects and the global control strategy can be left for later, when the civil engineering and installation of the accelerator are well under way. Even the computer may be an embarrassment if chosen too early. It is clearly advantageous to remain flexible on the choice of a computer, working on an arbitrary machine in high-level languages only, so that the resulting applications programs can be transported to a new computer if the one originally chosen becomes obsolete by the time the whole is assembled. Better still, one can aim for a fairly computer-independent scheme in which modern computers can coexist with old-fashioned ones.

The initial commissioning of an accelerator is a very important thing to plan well, but it should be understood that if this "bottom up" approach is developed for commissioning from the subsystem up to the whole, the same approach can be used for making changes or adding new subsystems to the accelerator subsequently. This is one of the factors which makes the difference between a good control system and a bad one.

\subsection{Open-ended Design}

This is probably the most difficult aspect of control system design. It is one thing to design a control system which will work with a given accelerator when it is switched on, and quite another to produce a system which is robust enough to weather changes in the technology, developments in the accelerator itself, and even expansion to an unimagined scale or combinations with machines whose control systems have been designed by other people. A complication is that the budget available for controls is usually adequate for the short-term purpose, but ingufficiently lavish to allow the use of grandiose generalized schemes that provide the open-endedness which will be needed over the long term. It is however important at the design stage to make the system modular in such a way that extra facilities can always be obtained by adding hardware, not by re-building the system.

For example, consider the requirement to control some extra devices. If each such extra device requires a certain amount of extra memory, and there is an absolute limit on the memory which can be installed on that particular computer, then there will arrive a time when it is impossible (not difficult, impossible) to add another device. This is due to bad design. A good system architecture would foresee this possibility and allow the problem to be solved by the 
purchase and installation of an extra computer at this point, or by replacement with a plug-compatible computer which offers more memory space.

Thus when doing the original design each major dimension of the system should be scrutinized for the conceivable limits to its expansion. The system should be so designed that the extra capacity can be bought for money, rather than by a fundamental re-design of the system. Such flexibility does not come free of cost, of course, but the extra cost of having it should be budgeted. The alternative is to have a system which works, but which needs to be re-designed and re-built if the requirements change.

The importance of being able to buy oneself out of a limitation extends not only to size, but also to speed. Data links should be so disposed that if extra bandwidth is necessary, it can be purchased by the installation of parallel channels or replacement of the links by more advanced systems. Here we should look for bottlenecks: if the link is faster, can the computer keep up? If not, is there a version of the computer with faster input/output, or can two computers be used?

Finally, it is useful when building a system which is to serve for many years to consider the possible system extensions. In this respect one should consider how, given the need and the resources, one could attach lines to external computers for performing large-scale computations, close control loops with the result of such computations, install diagnostic and even self-repairing facilities for critical elements, provide remote facilities through public networks, and so on. Many of these extensions may never be necessary, but a well-designed system should allow them to be added if the need should arise. That they will then cosit money is unimportant, for should the need arise, the budget for doing the work will certainly be available. 


\section{Problem 1}

Possible design parameters for a $20 \mathrm{~T}$ ring include the use of $300^{\prime}$ long dipoles with $B=10$ Tesla which occupy $80 \%$ of the ring's circumference. It is necessary, for quench protection purposes, to monitor a (differential) voltage, at a $60 \mathrm{~Hz}$ rate every 40 feet along the magnet. For diagnostic purposes, one desires to save in a circular buffer a two second long snapshot of these voltages (for the most recent two seconds).

1. Calculate the digitization rate per magnet and the settling time required of an $A / D$ converter, assuming that a single converter is used per magnet.

2. Suppose that the control system for this ring is totally centralized and that the results of the digitization need to be transmitted to distant control computers for monitoring purposes. Assuming that 16 -bit $A / D$ converters are used, and that the digitized results are transmitted over a single serial link, calculate the necessary bandwidth of the link in Megabits/second. Would an Ethernet link be a possible candidate for the transaission scheme?

3. Suppose that the control system is distributed and that the digitized voltages are stored in memory units near each magnet. How many bytes of memory are needed to store, locally, a two-second snapshot for the entire ring of dipoles? Assuming a cost of 0.1 cents per bit, what is the total memory cost?

4. What do you deduce from these calculations about the advisability of storing complete post-morten information centrally? What alternatives do you suggest? 


\section{Problem 2}

A system requires microprocessor-based units for two purposes: Application $A$ is an elaborate function generator unit which needs a 16-bit microprocessor and associated memory giving a unit cost of $\$ 1000$. Application $B$ is a simple sequencer which can be made with an 8-bit processor in a unit costing $\$ 600$. The system needs 10 function generators and 50 sequencers. The program for scheme B costs 2 man-months of skilled effort (say $\$ 5000$ ) but would be essentially free if the more powerful processor were used in this case also, since much better software support is available for 16-bit processors. How much more does it cost to use a homogeneous 16-bit system? What other factors can be given cost values to strengthen the case for a homogeneous system? 
A (paper) design of the control system for a small (fictitious) accelerator has resulted in the configuration below:

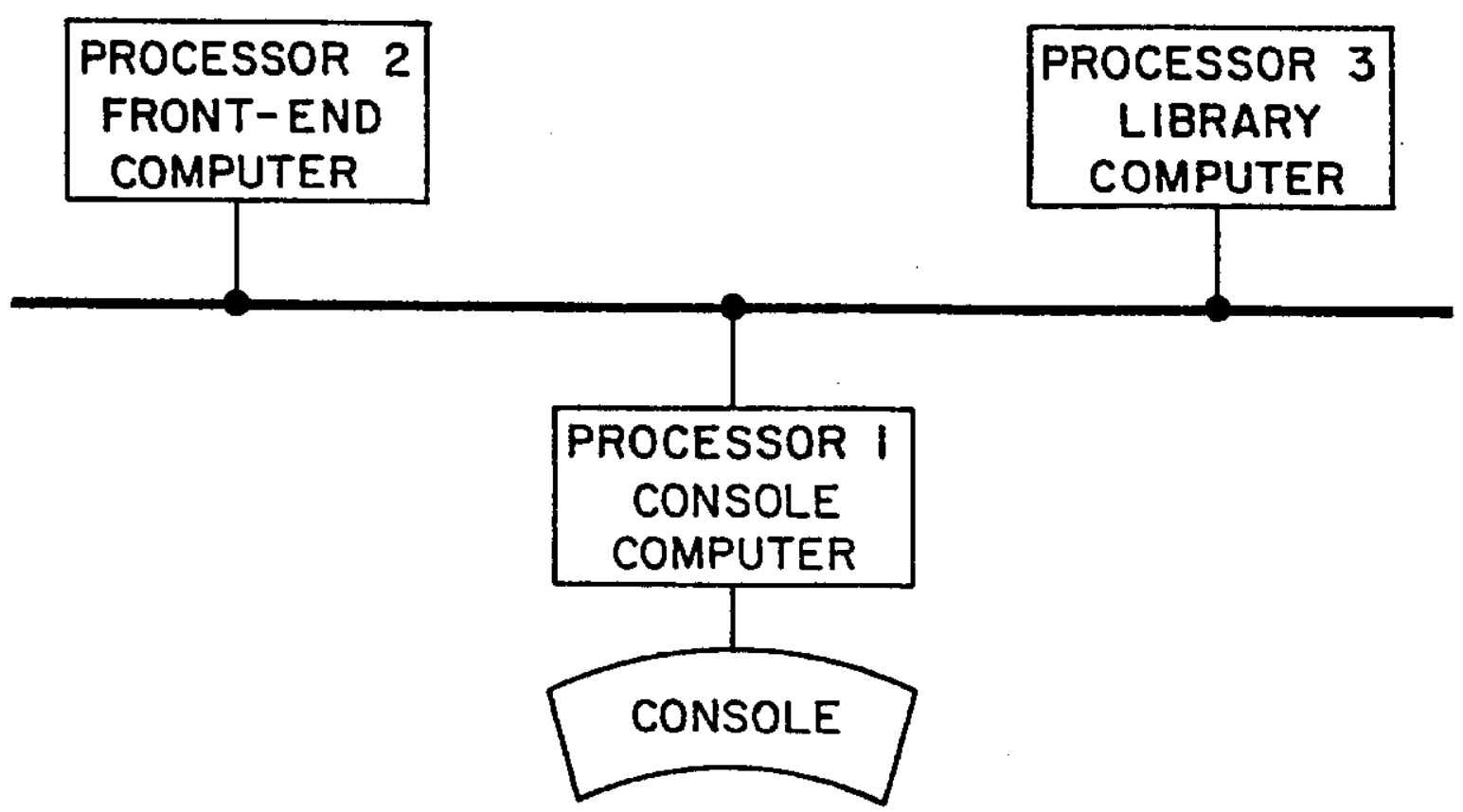

In this configuration the role of the console computer would be to service the console hardware, to execute the application programs (after retrieving them from the library), and to perform tolerance monitoring of selected accelerator devices. It is estimated that $64 \mathrm{~K}$ bytes of the processor's memory will be required to execute some of the large application programs which are anticipated. The tolerance monitoring routine is estimated to require an additional $16 \mathrm{~K}$ bytes of memory. The console hardware to be serviced by the console computer includes: (1) a keyboard and black and white video monitor, (2) a touch panel and (3) a high-resolution 512 × 512 pixel (i.e., picture element), 8-color display monitor which needs to be refreshed at a $30 \mathrm{~Hz}$ rate.

Suppose you are given the task of developing the detailed specifications for processor 1.

1. If the high-resolution monitor needed to be completely rewritten, every refresh cycle, by the console computer, what bandwidth would be required of an input/output channel 
on the console computer? How much internal memory would be required to hold the video image of the high resolution monitor?

2. The chores which the computer must execute (described above) suggest a multi-tasking operating system which typically requires approximately $32 \mathrm{~K}$ bytes of memory for its exclusive use. What would be a reasonable specification on the total amount of memory for the console computer? What would be a reasonable specification for the amount by which this memory could be expanded to accommodate future growth?

3. Discuss how both the memory requirement and the input/output bandwidth requirement for the console computer can be significantly reduced by using a high-resolution monitor which incorporates its own video refresh memory. What other requirements and specifications can be placed upon the console hardware to alleviate the memory and input/output bandwidth specifications on the computer?

4. It is often possible to reduce the software complexity of a single computer by replacing it with two processors and carefully redistributing the repertoire of tasks between the two processors. Suggest variations of Fig. 1 which would replace the computer with two or more computers. What are some of the advantages of this approach? Would the use of a single-task operating system be worth considering for one or more of the new processors? What are some of the disadvantages of this approach? Under what conditions would there be a significant increase in the amount of inter-processor communication in this approach? 


\section{References}

1. G. Baribaud et al., IEEE Trans. Nuc1. Sci., NS-26, 3272 (1979).

2. R.H. Flora and G.S. Tool, IEEE Trans. Nucl. Sci., NS-26, 3451 (1979).

3. M.A. Allen, M. Cornacchia, and M. Millich, IEEE Trans. Nucl. Sci., NS-26, 3287 (1979).

4. K. Endo and A. Ando, IEEE Trans. Nucl. Sci., NS-26, 3328 (1979).

5. W.H. Deluca, A.J. Maier, J.E. Michelsen, and C.A. Swoboda, IEEE Trans. Nucl. Sci., NS-20, 552 (1973).

6. IEEE Std. 448-1975, Inst. of Electrical and Electronic Engineers, New York.

7. P. Borrill, IEEE Micro, Vol. 1, No. 1, 84 (1981).

8. IEEE Std. 583-1975, Inst. of Electrical and Electronic Engineers, New York.

9. H. Verweij, CERN-EP Electronics Note 82-03 (1982).

10. STD Bus Technical Manual and Product Catalog, Pro-Log Corp., Monterrey, CA (1982).

11. D.B. Gustavson, IEEE Trans. Nucl. Sci., NS-27, 606 (1980).

42. IEEE Std. 696-1982, Inst. of Electrical and Electronic Engineers, 1982.

13. IEEE Sta. 796-1982, Inst. of Electrical and Electronic Engineers, 1982.

14. B. Wadsworth, IEEE Trans. Nucl. Sci., NS-27, 612 (1980).

15. FASTBUS, U.S. NIM Committee (1982).

16. S.R. Deiss, R.W. Downing, D.B. Gustavson, R.S. Larsen, C.A. Logg, and I. Paffrath, IEEE Trans. Nucl. Sci., NS-28, 2222 (1981).

17. A. Allison, IEEE Micro, Vol. 1, No. 1, 67 (1981).

18. K. Jones, Mini-Micro Systems, 18 (December, 1981).

19. H.S. Butler, B.L. Hartway, D.R. Machen, and T.M. Putman, IEEE Trans. Nucl. Sci., NS-18, 419 (1971). 
20. F. Beck, CERN-SPS 76-01 (1976).

21. P. Walich, IEEE Spectrum, Vol. 20, No. 1, 71 (1983).

22. K.J. Thurber and H.A. Freeman, Electronics, Vol. 55, No. 2, 93 (1982).

23. The Ethernet: A Local Area Network, Digital Equipment Corporation and Intel Corporation and Xerox Corporation (4980).

24. J.F. Shoch and J.A. Hupp, Compcon Spring 80, IEEE Computer Society, Los Alamitos, CA ( $(980)$.

25. IEEE Draft Std. P802, Inst. of Electrical and Electronic Engineers, 1982.

26. M. C. Crowley-Milliney and G. Shering, "The Nodal System for the SPS", CERN 78-07.

27. R.A. Belshe, V.P. Elisher, and V. Jacobson, IEEE Trans. Nucl. Sci., NS-22, 1036 (1975).

28. B. Kernighan and D. Ritchie, The $\subseteq$ Programming Language, (Englewood Cliffs, New Jersey: Prentice-Hall, Inc., 1978).

29. J. Altaber and R. Rausch, 1983 Particle Accelerator Conference, March 21-23, 1983; Santa Fe, New Mexico. 